\title{
Three Component Reaction: An Efficient Synthesis and Reactions of 3,4-Dihydropyrimidin-2(1H)-Ones and Thiones Using New Natural Catalyst
}

\author{
A. M. Elmaghraby, I. A. Mousa, A. A. Harb, and M. Y. Mahgoub \\ Department of Chemistry, Faculty of Science, South Valley University, Qena 83523, Egypt \\ Correspondence should be addressed to A. M. Elmaghraby; awatef_elmaghraby@yahoo.com
}

Received 28 May 2013; Accepted 1 July 2013

Academic Editors: S. Bellemin-Laponnaz, D. K. Chand, T. C. Dinadayalane, and J. Wu

Copyright (C) 2013 A. M. Elmaghraby et al. This is an open access article distributed under the Creative Commons Attribution License, which permits unrestricted use, distribution, and reproduction in any medium, provided the original work is properly cited.

Synthesis of 3,4-dihydropyrimidin-2(1H)-one and 3,4-dihydropyrimidin-2(1H)-thione derivatives from aldehydes, 1,3-dicarbonyl derivatives and urea or thiourea using granite and quartz as new, natural and reusable catalysts. Some of the 3,4-dihydropyrimidin$2(1 H)$-thione derivatives were used to prepare new heterocyclic compounds. The antimicrobial activity of selected examples of the synthesized compounds was tested and showed moderate activity.

\section{Introduction}

Aryl-3,4-dihydropyrimidines derivatives have recently received great attention because of their wide range of therapeutic and pharmacological properties, such as antiviral [1], antitumor, antibacterial and antifungal [2], anti-inflammatory [3], antihypertensive agents, and neuropeptide $\mathrm{Y}$ (NPY) antagonists [4]. Furthermore, these compounds have emerged as the integral backbones of several calcium-channel blockers [5]. Also, several alkaloids containing the dihydropyrimidine were isolated from marine sources, for example, of these are the batzelladine alkaloids, which are found to be potent HIVgp-120-CD4 inhibitors [6, 7].

In general, the classic Biginelli approach to 3,4-dihydropyrimidinones is based on the condensation of ethyl acetoacetate, aromatic aldehyde, and urea under strong acidic conditions; this suffers, however, from low yields of products, particularly in case of substituted aromatic and aliphatic aldehydes $[8,9]$. This problem has led to the development of multistep synthetic strategies that produce relatively higher yields, but lack the simplicity of the original one-pot-Biginelli protocol. Thus, the Biginelli reaction has received renewed interest from researchers interested in discovering milder and more efficient procedures that are applicable to a wide range of substituents in all three components and proceed in better yields. So, the one-potBiginelli protocol for 3,4-dihydropyrimidines synthesis was explored by varying all components and catalysts [10-18] in protic, aprotic solvents, and solvent free conditions [19] using either classical heating, microwave [20,21], ultrasound $[22,23]$, and visible light (100 W Lamp, THF) irradiations [24]. Also several improved procedures have been reported recently using not only acidic media such as Lewis acids, protic acids, and ionic liquids as promoters $[25,26]$ but also nonacidic substances such as baker's yeast [27], graphite [28], and iodine [29, 30]. Heterogeneous solid acids are used also; however, these are advantageous over conventional homogeneous acid catalysts as they can be easily recovered from the reaction mixture by simple filtration and can be reused after activation or without activation, thereby making the process economically viable [31]. Bakibaev and Filimonov [32] reported that piperidine as a base catalyst can promote the Biginelli protocol also, to afford the corresponding 3,4-dihydropyrimidines along with Hantzsch 1,4dihydropyridines which may form in spite of urea decomposition in the reaction media, releasing ammonia. We would like to propose a new naturally and very cheap catalysts granite and quartz for the synthesis of 3,4-dihydropyrimidinones and 3,4-dihydropyrimidenthiones, using one-pot-Biginelli protocol, in refluxing ethanol. 
TABLE 1: 3,4-Dihydropyrimidin-2(1H)ones (I).

\begin{tabular}{|c|c|c|c|c|c|c|c|c|}
\hline \multirow{2}{*}{ Entry } & \multirow{2}{*}{$\mathrm{R}_{1}$} & \multirow{2}{*}{$\mathrm{R}_{2}$} & \multicolumn{2}{|c|}{ Time (h) } & \multicolumn{2}{|c|}{ MP. [Reference] } & \multicolumn{2}{|c|}{ Yield $^{\mathrm{a}}$} \\
\hline & & & Quartz & Granite & Found & Reported & Quartz & Granite \\
\hline Ia & $\mathrm{C}_{6} \mathrm{H}_{5}$ & OEt & 3 & 3.5 & 201-202 & $200-202$ [33] & 68 & 64 \\
\hline Ib & $2-(\mathrm{OH})-\mathrm{C}_{6} \mathrm{H}_{4}$ & OEt & 3.5 & 5 & $202-204$ & 200-202 [34] & 62 & 60 \\
\hline Ic & $4-\left(\mathrm{OCH}_{3}\right)-\mathrm{C}_{6} \mathrm{H}_{4}$ & OEt & 3 & 3 & $199-200$ & $201-202[35]$ & 64 & 60 \\
\hline Id & $\mathrm{Ph}-\mathrm{CH}=\mathrm{CH}$ & OEt & 3 & 4 & $232-234$ & $232-235[36]$ & 58 & 55 \\
\hline Ie & $2,5-\left(\mathrm{OCH}_{3}\right)-\mathrm{C}_{6} \mathrm{H}_{3}$ & OEt & 4 & 5 & $210-212$ & $212-214$ [37] & 61 & 58 \\
\hline If & $3,4,5-\left(\mathrm{OCH}_{3}\right)-\mathrm{C}_{6} \mathrm{H}_{2}$ & OEt & 3 & 4.5 & $180-181$ & $180-182[25]$ & 60 & 56 \\
\hline Ig & 2-furyl & OEt & 3 & 4 & $205-206$ & $203-205[38]$ & 66 & 61 \\
\hline Ih & $2-(\mathrm{Cl})-\mathrm{C}_{6} \mathrm{H}_{4}$ & OEt & 3 & 4 & $214-215$ & $215-216$ [39] & 65 & 62 \\
\hline Ii & $4-\left(\mathrm{OCH}_{3}\right)-\mathrm{C}_{6} \mathrm{H}_{4}$ & $\mathrm{CH}_{3}$ & 3 & 4 & $165-167$ & $166-168[40]$ & 69 & 65 \\
\hline $\mathbf{I j}$ & $4-\mathrm{N}\left(\mathrm{CH}_{3}\right)_{2}-\mathrm{C}_{6} \mathrm{H}_{4}$ & OEt & 3 & 4 & $233-235$ & $230-232[25]$ & 62 & 58 \\
\hline Ik & $4-\left(\mathrm{CH}_{3}\right)-\mathrm{C}_{6} \mathrm{H}_{4}$ & OEt & 2 & 3 & $210-212$ & $214-215[35]$ & 65 & 62 \\
\hline Il & $2,6-(\mathrm{Cl})-\mathrm{C}_{6} \mathrm{H}_{3}$ & OEt & 4 & 4.5 & $302-303$ & $305[41]$ & 57 & 61 \\
\hline Im & 2-thienyl & OEt & 2 & 3 & $214-216$ & $215-217[42]$ & 66 & 63 \\
\hline In & $4-(\mathrm{F})-\mathrm{C}_{6} \mathrm{H}_{4}$ & OEt & 2 & 3 & $175-177$ & $175-177[43]$ & 71 & 68 \\
\hline Io & $3-\left(\mathrm{OCH}_{2} \mathrm{Ph}\right)-\mathrm{C}_{6} \mathrm{H}_{4}$ & OEt & 3 & 3.5 & $178-180$ & $\mathrm{New}$ & 65 & 62 \\
\hline Ip & 3- $\left(\mathrm{OCH}_{2} \mathrm{Ph}\right)-\mathrm{C}_{6} \mathrm{H}_{4}$ & $\mathrm{CH}_{3}$ & 3.5 & 4 & $192-194$ & New & 40 & 63 \\
\hline Iq & $2,3-\left(\mathrm{OCH}_{3}\right)-\mathrm{C}_{6} \mathrm{H}_{3}$ & OEt & 3 & 4 & $178-180$ & New & 63 & 60 \\
\hline
\end{tabular}

${ }^{\mathrm{a}}$ Isolated yield.

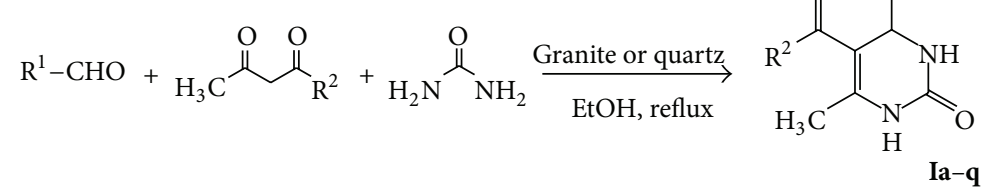

SCHEME 1

\section{Result and Discussion}

It is interesting to report that the one pot reaction of a mixture of benzaldehyde, ethyl acetoacetate, and urea in the presence of granite or quartz as a catalyst in refluxing ethanol resulted in the formation of 4-phenyl-3,4-dihydropyrimidinone Ia, Table 1 in $64 \%$ or $68 \%$ yield according to the catalyst (Scheme 1). In a similar way, urea was condensed smoothly with variety of aromatic or heterocyclic aldehydes and variety of 1,3-dicarbonyl compounds in the presence of granite or quartz in refluxing ethanol as one pot reaction to afford the corresponding 3,4-dihydropyrimidines Ib-q (Table 1) whose composition and structures were confirmed by elemental analysis, Mass, IR, and ${ }^{1} \mathrm{H}$ NMR spectra of the isolated products ( $c f$. experimental section).

On the other hand, carrying out of the above reaction using of 3-benzyloxybenzaldehyde, acetyl acetone, and urea in refluxing ethanol using granite as catalyst, the corresponding 5-acetyl-4-(3-(benzyloxy)phenyl)-6-methyl-3,4dihydropyrimidin-2(1H)-one Io was isolated. However, on carrying the above reaction using quartz as a catalyst, beside the proposed 3,4-dihydropyrimidinone Io, another product with molecular formula $\left(\mathrm{C}_{16} \mathrm{H}_{16} \mathrm{~N}_{4} \mathrm{O}_{2}\right), m / z=296$ was isolated from the reaction media in $25 \%$ yield. This product can be identified as 4-amino-6-(3-(benzyloxy)phenyl)-5,6dihydro-1,3,5-triazin-2(1H)-one III based on the analytical and the spectral data of the isolated product, which revealed the presence of characteristic stretching vibrations due to $\mathrm{NH}, \mathrm{NH}_{2}$, and amidic $\mathrm{CO}$ at $\nu=3450,3300$, and $1640 \mathrm{~cm}^{-1}$ regions, respectively, in the IR spectrum. Also, the ${ }^{1} \mathrm{H}-\mathrm{NMR}$ spectrum of the isolated product shows signals at $\delta=5.07$ $\left(\mathrm{s}, 2 \mathrm{H},-\mathrm{CH}_{2}-\right), 5.43(\mathrm{~s}, 1 \mathrm{H},-\mathrm{CH}-), 5.68\left(\mathrm{~s}, 2 \mathrm{H},-\mathrm{NH}_{2}\right)$, 6.70-7.46 (m, 9H, Ar), and 10.0 (s, 1H, NH) ppm. The ${ }^{13} \mathrm{C}-$ NMR spectrum of the isolated product shows signals at $\delta=51.2\left(\mathrm{CH}\right.$ aliphatic), $62.32\left(\mathrm{CH}_{2}\right.$ aliphatic $), 165.3(\mathrm{C}$ triazine ring), 190.4 ( $\mathrm{C}=\mathrm{O}$ amidic), and 111-160 (Benzene rings). This expectation is based on the observation that the 3-benzyloxybenzaldehyde condensed with two moles of urea to give the corresponding bis-ureide II as key intermediate which cyclized via elimination of $\mathrm{H}_{2} \mathrm{O}$ to give the extremely low yield triazine derivative III [44] (Scheme 2).

In generality of this process, various 1,3-diketones and aldehydes were reacted with thiourea in refluxing ethanol using granite or quartz as the reaction catalyst to give the corresponding 3,4-dihydropyrimidin-2(1H)-thione derivatives IV (Table 2) which their structures were confirmed on the bases of the analytical and spectral data of the isolated products (cf. experimental section) (Scheme 3 ). 


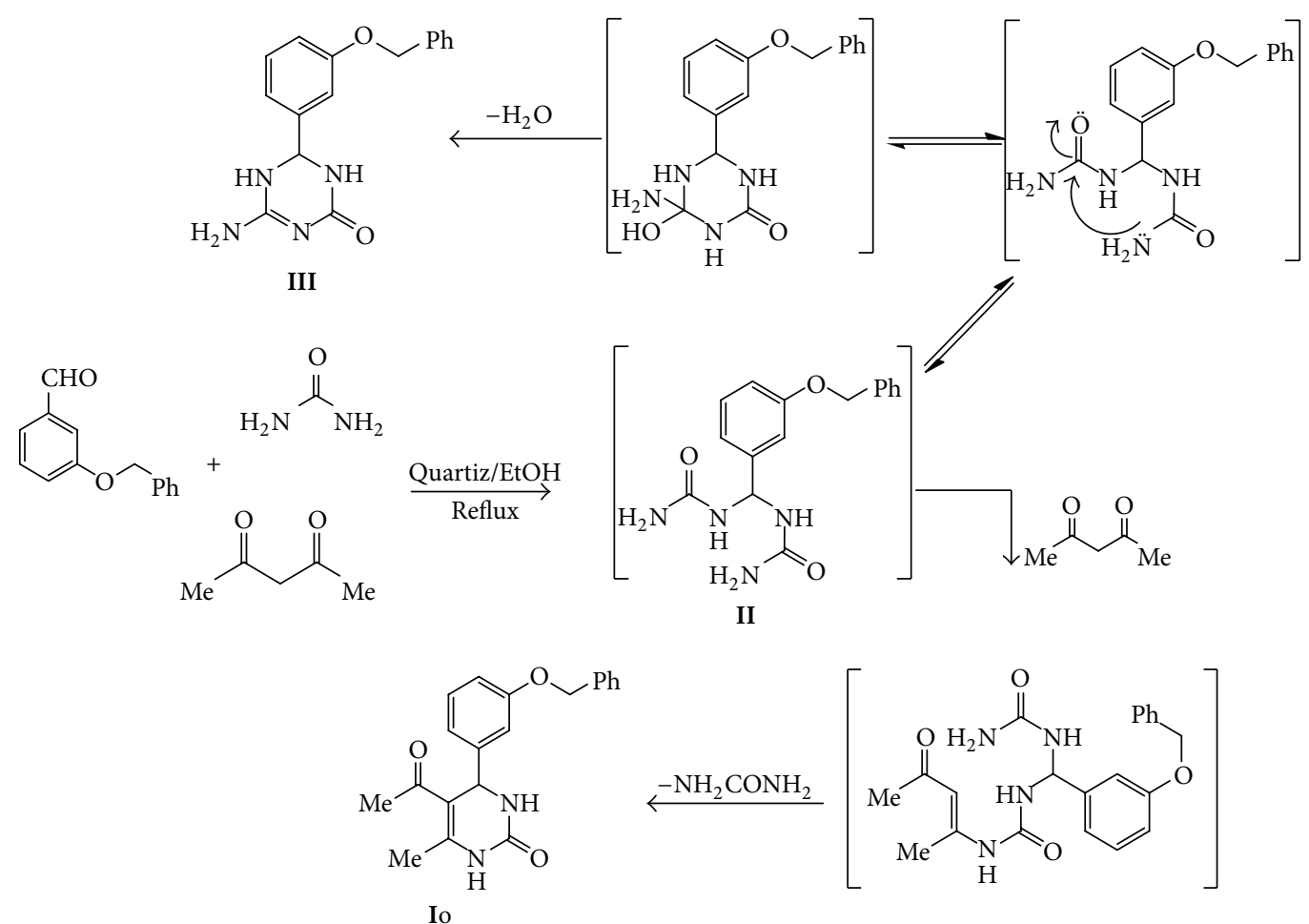

SCHEME 2

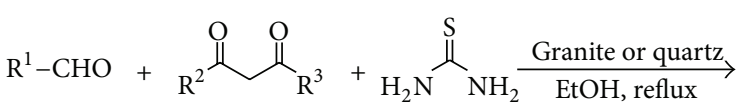<smiles>[R]C(=O)C1=C([R])NC(=S)NC1[R]</smiles>

IVa-h

SCHEME 3

On reading of the experimental results, we noted that aromatic aldehydes carrying either electron-donating or electron-withdrawing substituents reacted well under the reaction conditions to give the corresponding products in moderate to good yields high purity in case of granite or quartz. However, the obtained yields on using quartz are higher than granite either in case of urea or thiourea. This may be due to the high percentage of $\mathrm{SiO}_{2}$ in quartz. This procedure not only preserves the simplicity of the Biginelli reaction but also produces good yields of the products with high purity. Also, the catalyst was recovered by simple filtration and reused in subsequent reactions with consistent activity.

We can use the prepared 3,4-dihydropyrimidenthiones IVa-c to synthesize newly derivatives. Thus, heating of IVa-c with methyl iodide in dry acetone in the presence of anhydrous potassium carbonate afforded the $\mathrm{S}-\mathrm{CH}_{3}$ derivatives $\mathrm{Va}-\mathbf{c}$ which was confirmed by using elemental analysis and spectral data. The ${ }^{1} \mathrm{H}$ NMR illustrated the presence of singlet $\mathrm{S}-\mathrm{CH}_{3}$ protons.
On the other hand, heating of IVa-c in acetic anhydride afforded the corresponding 3-N-acetyl derivatives VIa-c. Structure VIc was deduced from elemental and spectral data. The mass spectrum showed the molecular ion peak at $\mathrm{m} / z$ $(\%)=378\left(\mathrm{M}^{+}, 48.03\right)$, for molecular formula $\mathrm{C}_{18} \mathrm{H}_{22} \mathrm{~N}_{2} \mathrm{O}_{5} \mathrm{~S}$. The ${ }^{1} \mathrm{H}$ NMR illustrated the presence singlet $\mathrm{N}-\mathrm{COCH}_{3}$ protons at $\delta=2.60 \mathrm{ppm}$, in addition to other singlet peaks at $\delta=2.27,3.67$, and 3.77 ppm for methyl and two methoxy groups, respectively, and the absence of the $\mathrm{NH}$ proton at $\delta=7.27$.

In the same time, we can use the same conditions to prepare VIa,b which was elucidated by correct elemental analysis and spectral data ( $c f$. experimental data). Also VIa-c was synthesized by the reaction of IVa-c with acetyl chloride in DMF (melting and mixed melting point) (Scheme 4).

In the same time, the pyrimidine derivatives VIIa,b can be synthesized via acetylation of the corresponding S$\mathrm{CH}_{3}$ derivatives Va,b using acetic anhydride. Also, it can be prepared via methylation of the $N$-acetyl derivatives VIa,b. Structures VIIa,b were elucidated by elemental analysis and 


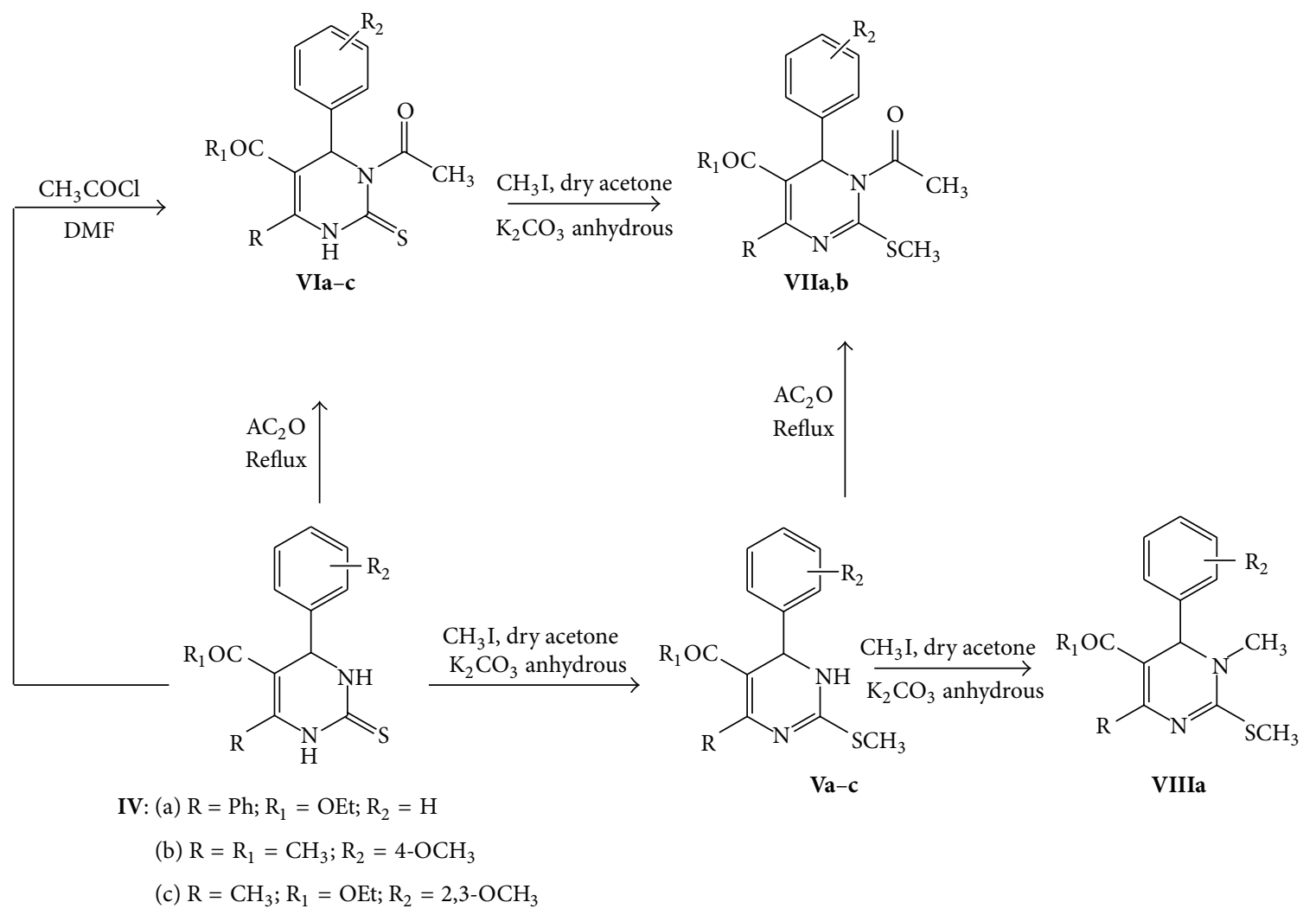

SCheme 4

TABLE 2: 3,4-Dihydropyrimidin-2(1H)-thiones (IV).

\begin{tabular}{|c|c|c|c|c|c|c|c|c|c|}
\hline \multirow{2}{*}{ Entry } & \multirow{2}{*}{$\mathrm{R}_{1}$} & \multirow{2}{*}{$\mathrm{R}_{2}$} & \multirow{2}{*}{$\mathrm{R}_{3}$} & \multicolumn{2}{|c|}{ Time (h) } & \multicolumn{2}{|c|}{ MP. [Reference] } & \multicolumn{2}{|c|}{ Yield $^{\mathrm{a}}$} \\
\hline & & & & Quartz & Granite & Found & Reported & Quartz & Granite \\
\hline IVa & $\mathrm{C}_{6} \mathrm{H}_{5}$ & OEt & $\mathrm{Ph}$ & 3 & 3 & $183-184$ & $183-185[45]$ & 63 & 60 \\
\hline IVb & $4-\left(\mathrm{OCH}_{3}\right)-\mathrm{C}_{6} \mathrm{H}_{4}$ & $\mathrm{CH}_{3}$ & $\mathrm{CH}_{3}$ & 3.5 & 4 & $181-182$ & $183-184[46]$ & 67 & 65 \\
\hline IVc & $2,3-\left(\mathrm{OCH}_{3}\right)-\mathrm{C}_{6} \mathrm{H}_{3}$ & OEt & $\mathrm{CH}_{3}$ & 3.5 & 4 & $181-183$ & New & 64 & 63 \\
\hline IVd & $4-\left(\mathrm{OCH}_{3}\right)-\mathrm{C}_{6} \mathrm{H}_{4}$ & OEt & $\mathrm{CH}_{3}$ & 3 & 4 & $152-153$ & $150-152[43]$ & 59 & 56 \\
\hline IVe & $2-(\mathrm{OH})-\mathrm{C}_{6} \mathrm{H}_{4}$ & OEt & $\mathrm{CH}_{3}$ & 3 & 4 & $210-211$ & $206-208$ [47] & 63 & 59 \\
\hline IVf & $2,6-(\mathrm{Cl})-\mathrm{C}_{6} \mathrm{H}_{3}$ & OEt & $\mathrm{CH}_{3}$ & 4 & 5 & $222-224$ & New & 55 & 60 \\
\hline IVg & $3-\left(\mathrm{OCH}_{2} \mathrm{Ph}\right)-\mathrm{C}_{6} \mathrm{H}_{4}$ & OEt & $\mathrm{CH}_{3}$ & 3 & 4 & $180-182$ & New & 66 & 60 \\
\hline IVh & $2,5-\left(\mathrm{OCH}_{3}\right)-\mathrm{C}_{6} \mathrm{H}_{3}$ & OEt & $\mathrm{CH}_{3}$ & 4.5 & 5 & $188-190$ & New & 64 & 60 \\
\hline
\end{tabular}

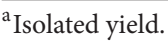

spectral data. The mass spectrum for VIIa showed the molecular ion peak at $m / z(\%)=394\left(\mathrm{M}^{+}, 12.51\right)$, while VIIb illustrated the molecular ion peak at $m / z(\%)=332\left(\mathrm{M}^{+}\right.$, 43.22). The ${ }^{1} \mathrm{H}$ NMR revealed the presence of singlet peak at $\delta=2.50 \mathrm{ppm}$ for $\mathrm{COCH}_{3}$ protons and the absence of the singlet peak at $\delta=7.27 \mathrm{ppm}$ for $\mathrm{NH}$ proton. Also IR spectrum showed the absence of $\mathrm{NH}$ peak (Scheme 4).

Methylation of Va was carried out in methyl iodide in DMF in the presence of $\mathrm{K}_{2} \mathrm{CO}_{3}$ anhydrous that yielded VIIIa which was confirmed by correct elemental analysis as well as spectral data. The ${ }^{1} \mathrm{H}$ NMR showed the absence of singlet peak at $\delta=7.27 \mathrm{ppm}$ for $\mathrm{NH}$ proton and the appearance of a singlet peak at $\delta=3.33 \mathrm{ppm}$ for $\mathrm{N}-\mathrm{CH}_{3}$ protons (Scheme 4 ).
Heating of IVa with ethylchloroacetate in ethanol and sodium acetate afforded ethyl 3-oxo-5,7-diphenyl-3,5,8,8atetrahydro- $2 \mathrm{H}$-thiazolo[3,2-a]pyrimidine-6-carboxylate Xa over the unisolated intermediate ethyl 2-(2-ethoxy-2-oxoethylthio)-4,6-diphenyl-1,6 dihydropyrimidine-5-carboxylate IXa as shown in elemental analysis as well as spectral data. The mass spectrum showed the molecular ion peak at $m / z(\%)=378\left(\mathrm{M}^{+}, 60.03\right)$ for molecular formula $\mathrm{C}_{21} \mathrm{H}_{18} \mathrm{~N}_{2} \mathrm{O}_{3} \mathrm{~S}$. The ${ }^{1} \mathrm{H}$ NMR revealed also the presence of one only ethyl ester group, at $\delta=0.85$ for $\mathrm{CH}_{3}$ protons $(\mathrm{t})$ and 3.85 for $\mathrm{CH}_{2}(\mathrm{q})$, and also the absence of $\mathrm{NH}$ proton at $\delta=7.27 \mathrm{ppm}$. The IR spectrum showed absorption bands at 1752,1675 , and $1589 \mathrm{~cm}^{-1}$ for carbonyl ester, amidic carbonyl 

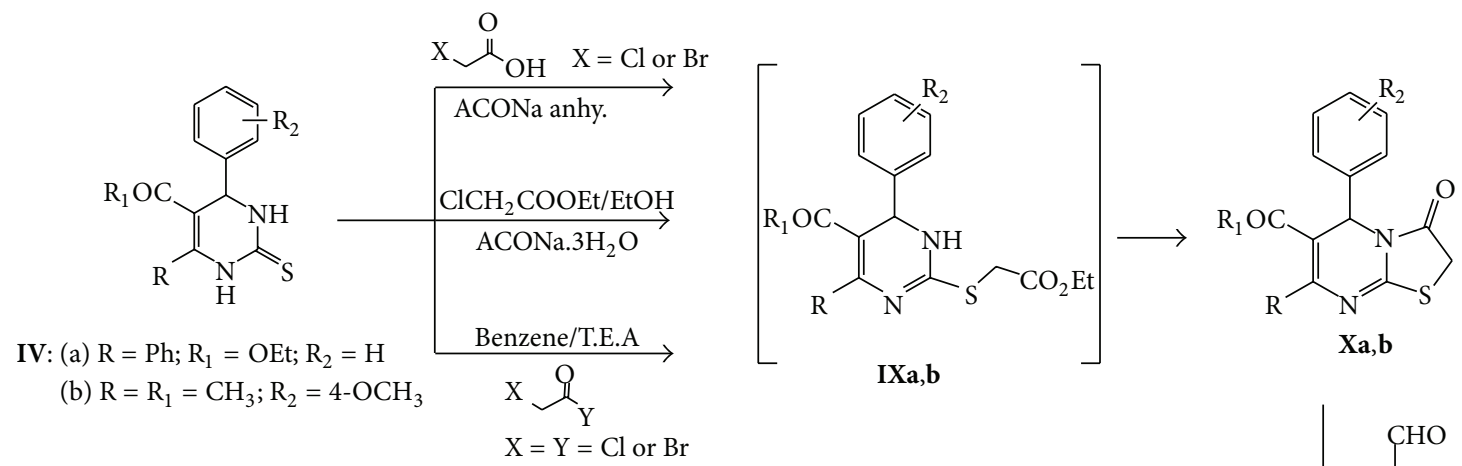

IXa,b
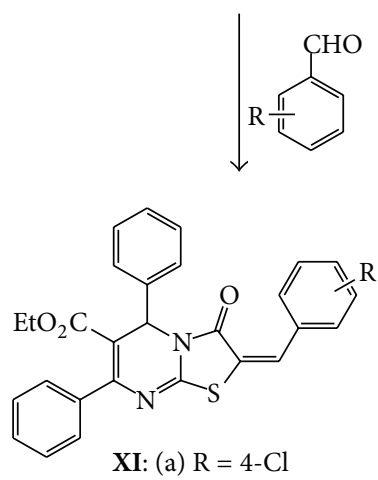

(b) $\mathrm{R}=2,3-\left(\mathrm{OCH}_{3}\right)$

(c) $\mathrm{R}=4-\mathrm{NO}_{2}$

SCHEME 5

groups, and $\mathrm{C}=\mathrm{N}$, respectively. Also, the isolated product Xa was obtained via the reaction of IVa with chloroacetyl chloride or bromoacetyl bromide in benzene and drops of triethylamine as catalyst. In the same time, compound Xa can be isolated from the reaction of IVa with chloroor bromoacetic acid in acetic acid and acetic anhydride mixture in presence of anhydrous sodium acetate. Similarly, compound $\mathbf{X b}$ was prepared from the reaction of $\mathbf{I V b}$ with ethylchloroacetate, chloroacetic acid, or chloroacetylchloride as shown in previous conditions (Scheme 5).

Compound $\mathbf{X a}$ was condensed with different aromatic aldehydes in refluxing ethanolic pipredine solution to give the corresponding arylidene derivatives XIa-c. Structures XIa-c were deduced from its elemental analysis and spectral data. The ${ }^{1} \mathrm{H}$ NMR showed the absence of singlet peak for $\mathrm{CH}_{2}$ protons at $\delta=3.88 \mathrm{ppm}$ and the appearance of singlet peak for $=\mathrm{CH}$ proton at $\delta=7.74 \mathrm{ppm}$ (Scheme 5).

Aiming to the synthesizing of thiazolopyrimidine XII, we refluxed $\mathbf{I V b}$ with chloroacetone in ethanolic piperidine solution. However the corresponding 1-(5-acetyl-6-(4-methoxyphenyl)-4-methyl-1,6-dihydropyrimidin-2-ylthio)propan-2-one XIII was formed which was identified by elemental analysis as well as spectral data. The mass spectrum showed the molecular ion peak at $m / z(\%)=332$ $\left(\mathrm{M}^{+}\right.$, 5.30) for molecular formula $\mathrm{C}_{17} \mathrm{H}_{20} \mathrm{~N}_{2} \mathrm{O}_{3} \mathrm{~S}$. The ${ }^{1} \mathrm{H}$ NMR confirmed the presence of only one $\mathrm{NH}$ proton at $\delta=7.11$ ppm and singlet peak at $\delta=2.46 \mathrm{ppm}$ due to $\mathrm{CH}_{2}$ protons (Scheme 6).

On the other hand, compound Va,b was reacted with thiosemicarbazide in refluxing ethanol to give the corresponding carbazide XIVa,b instead of the corresponding fused pyrimidinotriazoles XV and XVI. Structures XIVa,b were established by elemental analysis and spectral data where the mass spectrum showed the molecular ion peak at $m / z(\%)=395\left(\mathrm{M}^{+}, 24.13\right)$ for XIVa and at $m / z(\%)=333\left(\mathrm{M}^{+}\right.$, 12.18) for XIVb (Scheme 7).

On the other hand, refluxing of IVa,b in methyl alcohol in the presence of acetic acid and water $(4: 1: 1)$ afforded 3,4-dihydropyrimidinone derivatives XVIIa,b. Compound XVIIa was confirmed by compare mp. (Found) $=160^{\circ} \mathrm{C}$, mp. $($ Reported $)=158^{\circ} \mathrm{C}$ [48]. Also, compound XVIIb was established by compare $\mathrm{mp}$. (Found) $=164-166^{\circ} \mathrm{C} ; \mathrm{mp}$. $($ Reported $)=166-167^{\circ} \mathrm{C}[48]($ Scheme 8$)$.

\section{Antimicrobial Activity}

There are 5 compounds (III, IVg, IVf, IVh, and IVc) that were tested and showed promising positive antibacterial activity.

All the compounds showed activity against bacteria such as Staphylococcus aureus and Escherichia coli. The IVh \& IVc compounds showed positive antibacterial against $S$. aureus which are $14.5 \mathrm{~mm}$ and $14 \mathrm{~mm}$, respectively, which are 0.25 and $0.75 \mathrm{~mm}$ less than the zone around Streptomphenicol disc. This may be due the presence of sulfur atomand pyrimidine ring.

The other three most active compounds tested are compounds IVg, IVf, and III. The activity of these compounds against Staphylococcus aureus showed positive reactions, $12.75,12.5$, and $12 \mathrm{~mm}$ of inhibition zones, respectively, compared to the inhibition zone of antibiotic used, as indicated in 


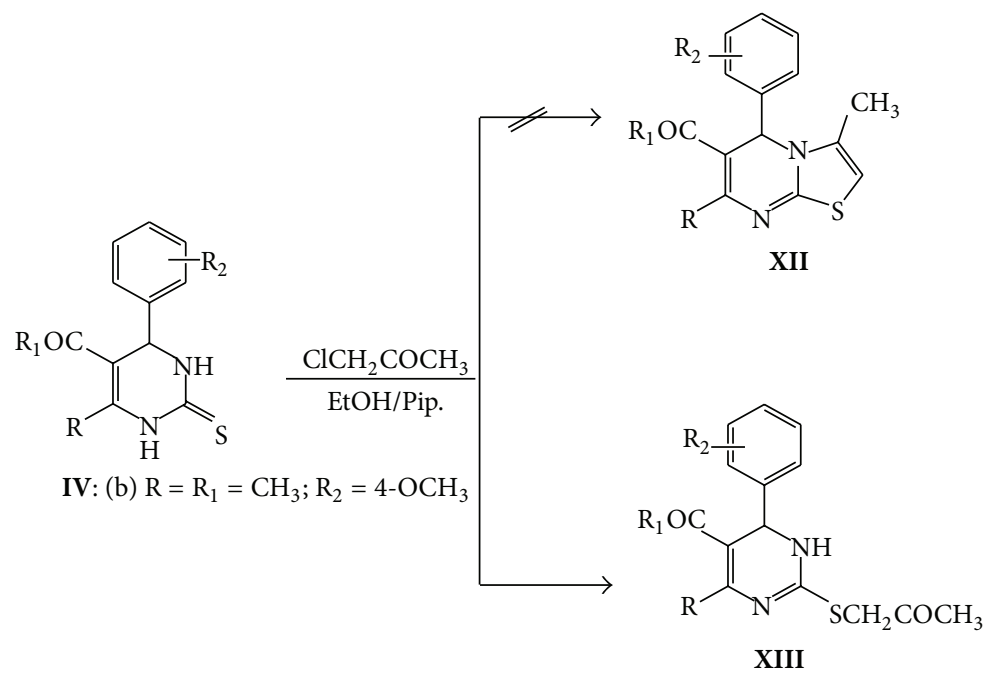

SCHEME 6

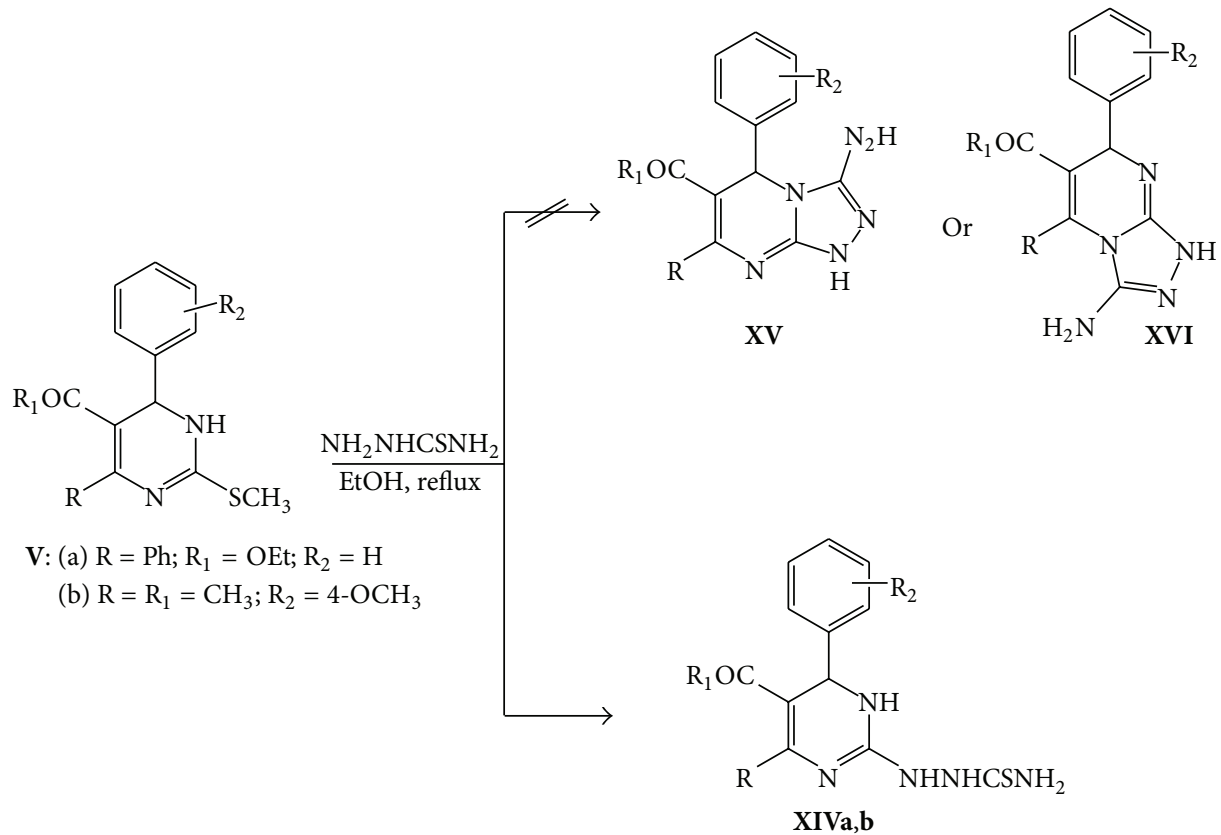

SCHEMe 7<smiles>[R]C(=O)C1=C([R])NC(=S)NC1c1ccccc1</smiles>

IV: (a) $\mathrm{R}=\mathrm{Ph} ; \mathrm{R}_{1}=\mathrm{OEt} ; \mathrm{R}_{2}=\mathrm{H}$ (b) $\mathrm{R}=\mathrm{R}_{1}=\mathrm{CH}_{3} ; \mathrm{R}_{2}=4-\mathrm{OCH}_{3}$<smiles>[R]C(=O)C1=C([R])NC(=O)NC1c1ccccc1</smiles>

XVIIa,b 
TABLE 3: Inhibition zone resulted from the effect of the antibiotic (Streptophenicol) and tested compounds on Escherichia coli and Staphylococcus aureus.

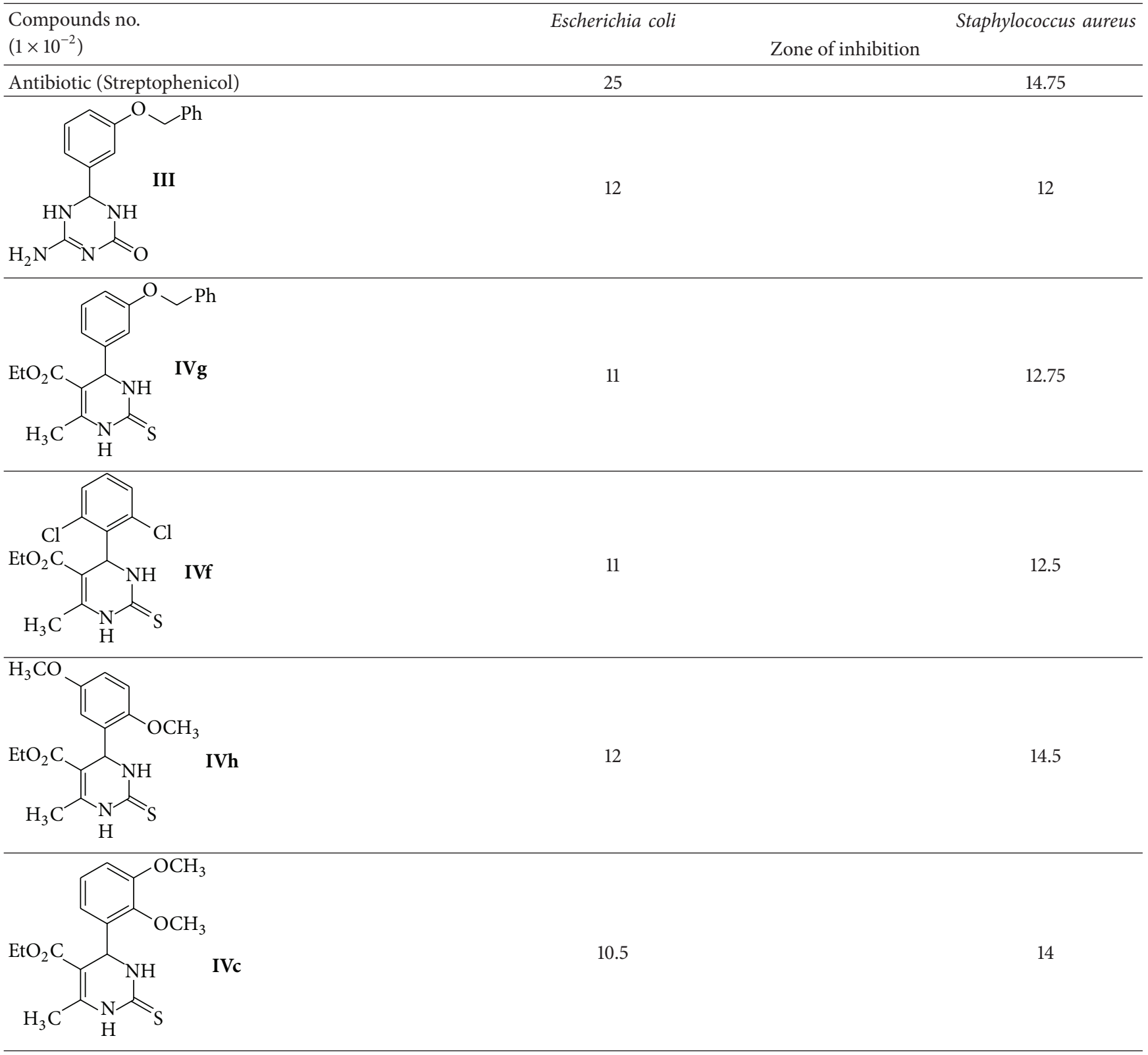

(Table 3); this may be due to sulfur atom, two chlorine atoms, and triazine ring, respectively.

All the compounds have approximately the same effect against Escherichia coli bacteria as indicated by the zone of inhibition (Table 3). In case of using these compounds as antimicrobial cytotoxicity, effect of these compounds must be examined.

\section{Conclusion}

In summary, we have found that quartz and granite are extremely useful and highly efficient new natural, solids for the synthesis of biologically potent aryl 3,4-dihydropyrimidines by means of three-component condensations of an aldehyde, 1,3-dicarbonyl compound, and urea or thiourea in a one-pot operation. This method is applicable to a wide range of substrates, including aromatic and heterocyclic aldehydes, and provides a variety of biologically relevant 3,4dihydropyrimidinones and 3,4-dihydropyrimidinthiones in high yields after short reaction times.

\section{Experimental Section}

5.1. General. All melting points were measured with a Gallenkamp apparatus. The IR spectra of samples were recorded in $\mathrm{KBr}$ via a Shimadzu FT-IR 8101 PC infrared spectrophotometer. ${ }^{1} \mathrm{H}$ NMR spectra were run at $300 \mathrm{MHz}$ and recorded in $\mathrm{CDCl}_{3} /[\mathrm{D} 6]$ DMSO using TMS as the internal standard. 
Chemical shifts were related to that of the solvent. Mass spectra were measured on a GCMS-QP1000 EX spectrometer at $70 \mathrm{eV}$. TLC was conducted on $0.25 \mathrm{~mm}$ precoated silica gel plates (60F-254). Elemental analyses were carried out at the Microanalytical Center of Cairo University, Giza, Egypt. The catalyst is ground until it became fine powder.

5.1.1. General Procedure for the Synthesis of the Newly 3,4Dihydropyrimidinones (Io,p,q) and 3,4-Dihydropyrimidinthiones (IVc,h,g,f). A mixture of aldehyde (1 mmol), 1,3dicarbonyl compounds $(1 \mathrm{mmol})$, urea or thiourea $(1 \mathrm{mmol})$, and granite or quartz $(0.5 \mathrm{~g})$ in ethanol $(15 \mathrm{~mL})$ was heated under reflux for the required time. After completion of the reaction as monitored by T.L.C., the reaction mixture was filtered to separate the catalyst. Keep the reaction mixture overnight. The solid product was filtered under suction then recrystallized from ethanol to afford pure product.

Ethyl-4-(3-(benzyloxy)phenyl)-6-methyl-2-oxo-1,2,3,4-tetrahydropyrimidine-5-carboxylate (Io). mp. $=178-180^{\circ} \mathrm{C}$. I.R $(\mathrm{KBr}): v=3300,3100,2950,1700,1630 \mathrm{~cm}^{-1} \cdot{ }^{1} \mathrm{H} \mathrm{NMR}$ $\left(300 \mathrm{MHz}, \mathrm{CDCl}_{3}\right): \delta=1.16\left(\mathrm{t}, J=7.2 \mathrm{~Hz}, 3 \mathrm{H},-\mathrm{O}-\mathrm{CH}_{2}-\right.$ $\left.\mathrm{CH}_{3}\right), 2.33\left(\mathrm{~s}, 3 \mathrm{H}, \mathrm{CH}_{3}\right), 4.08\left(\mathrm{q}, 2 \mathrm{H},-\mathrm{O}-\mathrm{CH}_{2}-\mathrm{CH}_{3}\right), 5.03$ (s, 2H, -O- $\left.\mathrm{CH}_{2}-\mathrm{Ph}\right), 5.37$ (s, 1H, - $\mathrm{CH}-$ ), 5.92 (s, 1H, -NH), 6.85-6.94 (m, 4H, Ar), 7.19-7.39 (m, 5H, Ar), 8.31 (s, 1H, -NH) ppm. Mass: $m / z(\%): 366\left(\mathrm{M}^{+}, 6.31\right), 275$ (22.72), 183 (24.94), 91 (100.0). $\mathrm{C}_{21} \mathrm{H}_{22} \mathrm{~N}_{2} \mathrm{O}_{4}$ (366): calculated, \%: C 68.84, H 6.05, N 7.65, O 17.47; found, \%: C 68.82, H 6.10, N 7.55 , O 17.46. Yield quartz (65\%), granite (62\%).

5-Acetyl-4-(3-(benzyloxy)phenyl)-6-methyl-3,4-dihydropyrimidin-2(1H)-one $(\mathrm{Ip}) . \mathrm{mp} .=192-194^{\circ} \mathrm{C} . \mathrm{I} \cdot \mathrm{R}(\mathrm{KBr}): \nu=3450$, 3200, 2950, 1640, $1590 \mathrm{~cm}^{-1}$. ${ }^{1} \mathrm{H}$ NMR (300 MHz, DMSO): $\delta=2.08\left(\mathrm{~s}, 3 \mathrm{H}, \mathrm{CH}_{3}\right), 2.27\left(\mathrm{~s}, 3 \mathrm{H},-\mathrm{COCH}_{3}\right), 5.05(\mathrm{~s}, 2 \mathrm{H}$, $-\mathrm{O}-\mathrm{CH}_{2}-\mathrm{Ph}$ ), 5.20 (s, 1H, -CH-), 6.81-6.92 (m, 4H, Ar), 7.21-7.45 (m, 5H, Ar), 7.81 (s, 1H, -NH), 9.17 (s, 1H, -NH) ppm. Mass: $m / z$ (\%): $336\left(\mathrm{M}^{+}, 1.6\right), 293$ (1.9), 245 (33), 153 (14.6), 91 (100.0). $\mathrm{C}_{20} \mathrm{H}_{20} \mathrm{~N}_{2} \mathrm{O}_{3}$ (336): calculated, \%: C 71.41, $\mathrm{H}$ 5.99, N 8.33, O 14.27; found, \%: C 71.40, H 6.0, N 8.30, O 14.21. Yield quartz (40\%), granite $(63 \%)$.

Ethyl-4-(2,3-dimethoxyphenyl)-6-methyl-2-oxo-1,2,3,4-tetrahydropyrimidine-5-carboxylate (Iq). mp. $=178-180^{\circ} \mathrm{C}$. I.R $(\mathrm{KBr}): v=3250,3100,2950,1700,1640 \mathrm{~cm}^{-1} .{ }^{1} \mathrm{H} \mathrm{NMR}$ $\left(300 \mathrm{MHz}, \mathrm{CDCl}_{3}\right): \delta=1.11\left(\mathrm{t}, J=7.2 \mathrm{~Hz}, 3 \mathrm{H},-\mathrm{O}-\mathrm{CH}_{2}-\right.$ $\left.\mathrm{CH}_{3}\right), 2.40\left(\mathrm{~s}, 3 \mathrm{H}, \mathrm{CH}_{3}\right), 3.87\left(\mathrm{~s}, 3 \mathrm{H},-\mathrm{OCH}_{3}\right), 3.92(\mathrm{~s}, 3 \mathrm{H}$, $\left.-\mathrm{OCH}_{3}\right), 4.06\left(\mathrm{q}, 2 \mathrm{H},-\mathrm{O}-\mathrm{CH}_{2}-\mathrm{CH}_{3}\right), 5.71$ (s, $\left.1 \mathrm{H},-\mathrm{CH}-\right)$, $5.72(\mathrm{~s}, 1 \mathrm{H},-\mathrm{NH}), 6.73(\mathrm{~d}, 1 \mathrm{H}, \mathrm{Ar}), 6.87$ (d, 1H, Ar), $6.98(\mathrm{t}$, $1 \mathrm{H}, \mathrm{Ar}), 7.27(\mathrm{~s}, 1 \mathrm{H},-\mathrm{NH})$ ppm. Mass: $m / z(\%): 320\left(\mathrm{M}^{+}\right.$, 13.7), 288 (100.0), 243 (55.2), 183 (94.9), 155 (68.9), 137 (60.1), 77 (47.3). $\mathrm{C}_{16} \mathrm{H}_{20} \mathrm{~N}_{2} \mathrm{O}_{5}$ (320): calculated, \%: C 59.99, H 6.29, N 8.74, O 24.97; found, \%: C 59.95, H 6.22, N 8.70, O 24.99. Yield quartz (63\%), granite (60\%).

Ethyl-4-(3-(benzyloxy)phenyl)-6-methyl-2-thioxo-1,2,3,4-tetrahydropyrimidine-5-carboxylate $(\mathbf{I V g}) . \mathrm{mp} .=180-182^{\circ} \mathrm{C}$. I.R $(\mathrm{KBr}): v=3400,3150,2950,1650 \mathrm{~cm}^{-1} .{ }^{1} \mathrm{H}$ NMR $(300 \mathrm{MHz}$, DMSO): $\delta=1.10\left(\mathrm{t}, J=7.2 \mathrm{~Hz}, 3 \mathrm{H},-\mathrm{O}-\mathrm{CH}_{2}-\mathrm{CH}_{3}\right)$, $2.27\left(\mathrm{~s}, 3 \mathrm{H}, \mathrm{CH}_{3}\right), 4.01$ (q, $\left.2 \mathrm{H},-\mathrm{O}-\mathrm{CH}_{2}-\mathrm{CH}_{3}\right), 5.05$ (s,
$2 \mathrm{H},-\mathrm{O}-\mathrm{CH}_{2}-\mathrm{Ph}$ ), 5.15 (s, 1H, $-\mathrm{CH}-$ ), 6.80 (m, 4H, Ar), 7.23-7.42 (m, 5H, Ar), 9.60 (s, 1H, -NH), 10.30 (s, 1H, -NH) ppm. Mass: $m / z(\%)$ : $382\left(\mathrm{M}^{+}, 17.6\right), 199$ (12.2), 91 (100.0). $\mathrm{C}_{21} \mathrm{H}_{22} \mathrm{~N}_{2} \mathrm{O}_{3} \mathrm{~S}$ (382): calculated, \%: C 65.95, H 5.80, N 7.32, O 12.55, S 8.38; found, \%: C 65.89, H 5.60, N 7.35, O 17.46, S 8.36. Yield quartz $(66 \%)$, granite $(60 \%)$.

Ethyl-4-(2,3-dimethoxyphenyl)-6-methyl-2-thioxo-1,2,3,4-tetrahydropyrimidine-5-carboxylate $(\mathrm{IVc}) . \mathrm{mp} .=181-183^{\circ} \mathrm{C}$. I.R (KBr): $\nu=3200,3100,2950,1705 \mathrm{~cm}^{-1} .{ }^{1} \mathrm{H}$ NMR $(300 \mathrm{MHz}$, $\left.\mathrm{CDCl}_{3}\right): \delta=1.10\left(\mathrm{t}, J=7.2 \mathrm{~Hz}, 3 \mathrm{H},-\mathrm{O}-\mathrm{CH}_{2}-\mathrm{CH}_{3}\right), 2.42(\mathrm{~s}$, $\left.3 \mathrm{H}, \mathrm{CH}_{3}\right), 3.86\left(\mathrm{~s}, 3 \mathrm{H},-\mathrm{OCH}_{3}\right), 3.93\left(\mathrm{~s}, 3 \mathrm{H},-\mathrm{OCH}_{3}\right), 4.05$ $\left(\mathrm{q}, 2 \mathrm{H},-\mathrm{O}-\mathrm{CH}_{2}-\mathrm{CH}_{3}\right), 5.71(\mathrm{~s}, 1 \mathrm{H},-\mathrm{CH}-), 6.68$ (d, $\left.1 \mathrm{H}, \mathrm{Ar}\right)$, 6.88 (d, 1H, Ar), 6.99 (t, 1H, Ar), $7.26(\mathrm{~s}, 1 \mathrm{H},-\mathrm{NH}), 8.10$ (s, $1 \mathrm{H},-\mathrm{NH})$ ppm. Mass: $m / z(\%): 336\left(\mathrm{M}^{+}, 76.4\right), 305$ (86.1), 289 (81.5), 263 (100.0), 199 (87.7), 171 (63.3), 153 (31.5), 77 (44.6). $\mathrm{C}_{16} \mathrm{H}_{20} \mathrm{~N}_{2} \mathrm{O}_{4} \mathrm{~S}$ (336): calculated, \%: C 57.12, $\mathrm{H}$ 5.99, N 8.33, O 19.02, S 9.53; found, \%: C 57.13, H 5.96, N 8.35, O 19.06, S 9.51. Yield quartz (64\%), granite (63\%).

Ethyl-4-(2,5-dimethoxyphenyl)-6-methyl-2-thioxo-1,2,3,4-tetrahydropyrimidine-5-carboxylate (IVh). mp. $=188-190^{\circ} \mathrm{C}$. I.R $(\mathrm{KBr}): \nu=3200,3100,2940,1700 \mathrm{~cm}^{-1} \cdot{ }^{1} \mathrm{H}$ NMR $(300 \mathrm{MHz}$, DMSO): $\delta=1.05\left(\mathrm{t}, J=8.2 \mathrm{~Hz}, 3 \mathrm{H},-\mathrm{O}-\mathrm{CH}_{2}-\mathrm{CH}_{3}\right), 2.27(\mathrm{~s}$, $\left.3 \mathrm{H}, \mathrm{CH}_{3}\right), 3.65\left(\mathrm{~s}, 3 \mathrm{H},-\mathrm{OCH}_{3}\right), 3.72\left(\mathrm{~s}, 3 \mathrm{H},-\mathrm{OCH}_{3}\right), 3.96$ (q, 2H, -O- $\mathrm{CH}_{2}-\mathrm{CH}_{3}$ ), 5.44 (s, 1H, -CH-), 6.57 (s, 1H, Ar), 6.83 (d, 1H, Ar), 6.94 (d, 1H, Ar), 9.24 (s, 1H, -NH), 10.24 (s, $1 \mathrm{H},-\mathrm{NH})$ ppm. Mass: $m / z(\%): 338\left(\mathrm{M}^{+}, 10.97\right), 279(10.49)$, 256 (19.23), 166 (45.38), 149 (100.0), 105 (28.13), 69 (90.57). $\mathrm{C}_{16} \mathrm{H}_{20} \mathrm{~N}_{2} \mathrm{O}_{4} \mathrm{~S}$ (336): calculated, \%: C 57.12, H 5.99, N 8.33, O 19.02, S 9.53; found, \%: C 57.14, H 5.96, N 8.30, O 19.04, S 9.56. Yield quartz (64\%), granite $(60 \%)$.

Ethyl-4-(2,6-dichlorophenyl)-6-methyl-2-thioxo-1,2,3,4-tetrahydropyrimidine-5-carboxylate (IVf). $\mathrm{mp} .=222-224^{\circ} \mathrm{C}$. I.R $(\mathrm{KBr}): v=3150,3000,2900,1750,1640 \mathrm{~cm}^{-1} .{ }^{1} \mathrm{H} \mathrm{NMR}$ $\left(300 \mathrm{MHz}, \mathrm{CDCl}_{3}\right): \delta=0.94\left(\mathrm{t}, J=6.6 \mathrm{~Hz}, 3 \mathrm{H},-\mathrm{O}-\mathrm{CH}_{2}-\right.$ $\mathrm{CH}_{3}$ ), 2.23 (s, 3H, $\mathrm{CH}_{3}$ ), 3.91 (q, $\left.2 \mathrm{H},-\mathrm{O}-\mathrm{CH}_{2}-\mathrm{CH}_{3}\right), 6.28$ (s, 1H, -CH-), 7.05-7.23 (m, 3H, Ar), 7.94 (s, 1H, -NH), 8.32 (s, $1 \mathrm{H},-\mathrm{NH})$ ppm. Mass: $m / z(\%): 344\left(\mathrm{M}^{+}, 23.9\right)$, $348\left(\mathrm{M}^{+4}, 7.6\right), 315$ (34.5), 199 (100.0), 171 (36.4), 153 (16.2). $\mathrm{C}_{14} \mathrm{H}_{14} \mathrm{Cl}_{2} \mathrm{~N}_{2} \mathrm{O}_{2} \mathrm{~S}$ (344): calculated, \%: C 48.70, $\mathrm{H} 4.09, \mathrm{Cl}$ 20.54, N 8.11, O 9.27, S 9.29; found, \%: C 48.72, $\mathrm{H} \mathrm{4.04,} \mathrm{Cl}$ 20.50, N 8.13, O 9.29, S 9.30. Yield quartz (55\%), granite $(60 \%)$.

4-Amino-6-(3-(benzyloxy)phenyl)-5,6-dihydro-1,3,5-triazin2(1H)-one (III). mp. $=172-174^{\circ} \mathrm{C} \mathrm{I} . \mathrm{R}(\mathrm{KBr}): v=3450,3300$, $1640 \mathrm{~cm}^{-1}$. ${ }^{1} \mathrm{H}$ NMR (300 MHz, DMSO): $\delta=5.07(\mathrm{~s}, 2 \mathrm{H}$, $\left.-\mathrm{CH}_{2}-\right), 5.43(\mathrm{~s}, 1 \mathrm{H},-\mathrm{CH}-), 5.68\left(\mathrm{~s}, 2 \mathrm{H},-\mathrm{NH}_{2}\right), 6.70-6.92$ (m, 4H, Ar), 6.98 (s, 1H, NH), 7.23-7.46 (m, 5H, Ar), 10.0 (s, $1 \mathrm{H}, \mathrm{NH}) \mathrm{ppm}$. The ${ }^{13} \mathrm{C}-\mathrm{NMR}(300 \mathrm{MHz}, \mathrm{DMSO}) \delta=51.2$ ( $\mathrm{CH}$ aliphatic), $62.32\left(\mathrm{CH}_{2}\right.$ aliphatic), 165.3 ( $\mathrm{C}$ triazine ring), 190.4 ( $\mathrm{C}=\mathrm{O}$ amidic), 111-160 (benzene rings). Mass: $\mathrm{m} / \mathrm{z}$ (\%): 296 ( $\left.\mathrm{M}^{+}, 54.58\right), 294$ (82.76), 253 (57.20), 227 (63.30), 203 (100.0), 182 (31.01), 171 (55.77), 131 (99.09), 104 (29.73). $\mathrm{C}_{16} \mathrm{H}_{16} \mathrm{~N}_{4} \mathrm{O}_{2}$ (296): calculated, \%: C 64.85, H 5.44, N 18.91, O 10.80; found, \%: C 64.82, H 5.40, N 18.93, O 10.82. Yield quartz (25\%). 
5.1.2. General Procedure of Methylation of Compounds IVac. A mixture of IVa-c $(0.005 \mathrm{~mol})$ and methyl iodide $(0.005 \mathrm{~mol})$ was dissolved in dry acetone in the presence of pot. Carbonate anhydrous was refluxed in water bath for 5 hours. The reaction mixture was filtered on hot then kept for overnight. The formed solid was filtered off and crystallized from an appropriate solvent to give Va,b,c.

Ethyl-2-(methylthio)-4,6-diphenyl-1,6-dihydropyrimidine-5carboxylate (Va). The formed solid was crystallized from petroleum ether/benzene $2: 1 ; \mathrm{mp} .=158-160^{\circ} \mathrm{C}$; I.R $(\mathrm{KBr})$ : $\nu=3280,2982,2807,1676,1614,1493 \mathrm{~cm}^{-1} ;{ }^{1} \mathrm{H} \mathrm{NMR}$ $\left(300 \mathrm{MHz}, \mathrm{CDCl}_{3}\right): \delta=0.87\left(\mathrm{t}, J=6.9 \mathrm{~Hz}, 3 \mathrm{H},-\mathrm{O}-\mathrm{CH}_{2}-\right.$ $\mathrm{CH}_{3}$ ), 2.49 (s, 3H, $-\mathrm{SCH}_{3}$ ), 3.86 (q, $\left.2 \mathrm{H},-\mathrm{O}-\mathrm{CH}_{2}-\mathrm{CH}_{3}\right), 5.74$ (s, 1H, -CH-), 7.27 (s, 1H, NH), 7.28-7.48 (m, 10H, Ar) ppm; mass: $m / z$ (\%): $352\left(\mathrm{M}^{+}, 23.35\right), 337$ (33.58), 323 (68.32), 275 (100.0), 77 (25.52); $\mathrm{C}_{20} \mathrm{H}_{20} \mathrm{~N}_{2} \mathrm{O}_{2} \mathrm{~S}$ (352): calculated, \%: C 68.16, H 5.72, N 7.95, O 9.08, S 9.10; Found, \%: C 68.13, H 5.75, N 7.97, O 9.10, S 9.12; yield (77\%).

1-(6-(4-Methoxyphenyl)-4-methyl-2-(methylthio)-1,6-dihydropyrimidin-5-yl)ethanone (Vb). The formed solid was crystallized from petroleum ether/ethanol $1: 1$; mp. $=126-$ $128^{\circ} \mathrm{C}$; I.R $(\mathrm{KBr}): v=3285,2950,2928,1639,1594 \mathrm{~cm}^{-1} ;{ }^{1} \mathrm{H}$ NMR (300 MHz, DMSO): $\delta=2.09\left(\mathrm{~s}, 3 \mathrm{H},-\mathrm{CH}_{3}\right), 2.23(\mathrm{~s}$, $\left.3 \mathrm{H},-\mathrm{SCH}_{3}\right), 2.28\left(\mathrm{~s}, 3 \mathrm{H},-\mathrm{COCH}_{3}\right), 3.70\left(\mathrm{~s}, 3 \mathrm{H},-\mathrm{OCH}_{3}\right)$, 5.54 (s, 1H, -CH-), 6.82-7.15 (d, d, 4H, Ar), 9.57 (s, 1H, -NH) ppm; mass: $m / z$ (\%): $288\left(\mathrm{M}^{+}, 29.40\right), 250$ (22.10), 183 (19.10), 73 (67.60), 57 (100.0); $\mathrm{C}_{15} \mathrm{H}_{18} \mathrm{~N}_{2} \mathrm{O}_{2} \mathrm{~S}$ (290): calculated, \%: C 62.04, H 6.25, N 9.65, O 11.02, S 11.04; found, \%: C 62.03, H $6.26, \mathrm{~N} 9.65, \mathrm{O} 11.04, \mathrm{~S} 11.03$; yield (86\%).

Ethyl-6-(2,3-dimethoxyphenyl)-4-methyl-2-(methylthio)-1,6dihydropyrimidine-5-carboxylate $(\mathrm{Vc})$. The solid product was crystallized from petroleum ether $(60-80) ; \mathrm{mp} .=140^{\circ} \mathrm{C}$; I.R $(\mathrm{KBr}): \nu=3321,2935,2832,1706,1669,1594 \mathrm{~cm}^{-1} ;{ }^{1} \mathrm{H}$ NMR (300 MHz, DMSO): $\delta=1.13(\mathrm{t}, J=7.5 \mathrm{~Hz}, 3 \mathrm{H}$, $\left.\mathrm{CH}_{3} \mathrm{CH}_{2} \mathrm{O}-\right), 2.39\left(\mathrm{~s}, 3 \mathrm{H},-\mathrm{CH}_{3}\right), 2.45$ (s, 3H, $\left.-\mathrm{SCH}_{3}\right)$, $3.87\left(\mathrm{~s}, 3 \mathrm{H},-\mathrm{OCH}_{3}\right), 3.93\left(\mathrm{~s}, 3 \mathrm{H},-\mathrm{OCH}_{3}\right), 4.06(\mathrm{q}, 2 \mathrm{H}$, $\left.\mathrm{CH}_{3} \mathrm{CH}_{2} \mathrm{O}-\right), 5.85(\mathrm{~s}, 1 \mathrm{H},-\mathrm{CH}), 6.77-7.02(\mathrm{~m}, 3 \mathrm{H}, \mathrm{Ar})$, 7.27 (s, $1 \mathrm{H},-\mathrm{NH})$ ppm; mass: $m / z(\%): 350\left(\mathrm{M}^{+}, 20.56\right)$, 335 (36.97), 321 (64.29), 303 (40.44), 213 (100.0), 77 (23.14); $\mathrm{C}_{17} \mathrm{H}_{22} \mathrm{~N}_{2} \mathrm{O}_{4} \mathrm{~S}$ (350): calculated, \%: C 58.27, H 6.33, N 7.99, O 18.26, S 9.15; found, \%: C 58.26, H 6.33, N 7.97, O 18.27, S 9.16; yield $(74 \%)$.

\subsubsection{Acylation of Compounds IVa-c}

Method (A). A mixture of IVa-c $(0.005 \mathrm{~mol})$ and acetyl chloride $(0.01 \mathrm{~mol})$ was refluxed in DMF $(15 \mathrm{~mL})$ as a solvent containing ( 5 drops) of triethylamine (TEA) for 1 hour and then stirred at room temperature for overnight, and then the solution was poured into ice with vigorous stirring, and then the solid product was filtered off and recrystallized from suitable solvent to afford compounds VIa,b,c.

Method (B). A solution of IVa-c $(0.01 \mathrm{~mol})$ in $15 \mathrm{~mL}$ of acetic anhydride was heated under reflux for 1.30 hour. The solution was then poured into $150 \mathrm{~mL}$ of ice-water and stirred for several hours until crystallization was complete. The precipitate was filtered and crystallized from suitable solvent to afford compounds VIa,b,c.

Ethyl 3-acetyl-4-(2,3-dimethoxyphenyl)-6-methyl-2-thioxo-1, 2,3,4-tetrahydropyrimidine-5-carboxylate (VIc). The solid product was recrystallized from ethanol; method $(A)$ : yield (81\%). method (B): yield (78\%); mp. $=186^{\circ} \mathrm{C}$; I.R (KBr): $v=3237,2996,2944,2837,1702,1671 \mathrm{~cm}^{-1} ;{ }^{1} \mathrm{H} \mathrm{NMR}$ (300 MHz, DMSO): $\delta=1.17\left(\mathrm{t}, J=6.6 \mathrm{~Hz}, 3 \mathrm{H}, \mathrm{CH}_{3} \mathrm{CH}_{2} \mathrm{O}-\right.$ ), $2.27\left(\mathrm{~s}, 3 \mathrm{H},-\mathrm{CH}_{3}\right), 2.60\left(\mathrm{~s}, 3 \mathrm{H},-\mathrm{COCH}_{3}\right), 3.67(\mathrm{~s}, 3 \mathrm{H}$, $\left.-\mathrm{OCH}_{3}\right), 3.77$ (s, 3H, $\left.-\mathrm{OCH}_{3}\right), 4.08$ (q, $\left.2 \mathrm{H}, \mathrm{CH}_{3} \mathrm{CH}_{2} \mathrm{O}-\right)$, $5.50(\mathrm{~s}, 1 \mathrm{H},-\mathrm{CH}), 6.68-7.0(\mathrm{~m}, 3 \mathrm{H}, \mathrm{Ar}), 11.6(\mathrm{~s}, 1 \mathrm{H},-\mathrm{NH})$ ppm; mass: $m / z(\%): 378\left(\mathrm{M}^{+}, 48.03\right), 335$ (96.81), 289 (100.0), 263 (45.57), 199 (33.62), 77 (22.44); $\mathrm{C}_{18} \mathrm{H}_{22} \mathrm{~N}_{2} \mathrm{O}_{5} \mathrm{~S}$ (378): calculated, \%: C 57.13, H 5.86, N 7.40, O 21.14, S 8.47; found, \%: C 57.14, H 5.85, N 7.41, O 21.13, S 8.45.

Ethyl 3-acetyl-4,6-diphenyl-2-thioxo-1,2,3,4-tetrahydropyrimidine-5-carboxylate (VIa). The solid product was recrystallized from benzene; method $(A)$ : yield (80\%); method $(B)$ : yield $(85 \%) . \mathrm{mp} .=136^{\circ} \mathrm{C}$; I.R $(\mathrm{KBr}): v=3215,2986,1642$, 1599, $1494 \mathrm{~cm}^{-1}$; mass: $m / z(\%): 380\left(\mathrm{M}^{+}, 37.39\right), 337$ (100.0), 307 (25.7), 265 (57.72), 104 (65.03); $\mathrm{C}_{21} \mathrm{H}_{20} \mathrm{~N}_{2} \mathrm{O}_{3} \mathrm{~S}$ (380): calculated, \%: C 66.29, H 5.30, N 7.36, O 12.62, S 8.43; found, \%: C 66.28, H 5.31, N 7.35, O 12.63, S 8.42.

1,1' -(6-(4-Methoxyphenyl)-4-methyl-2-thioxo-2,3-dihydropyrimidine-1,5(6H)-diyl)diethanone (VIb). The solid product was recrystallized from ethanol; method $(A)$ : yield $(65 \%)$; method (B): yield (70\%). mp. $=130^{\circ} \mathrm{C}$; I.R $(\mathrm{KBr}): \nu=3243$, 2962, 1698, 1609, $1509 \mathrm{~cm}^{-1}$.

5.1.4. Synthesis of Compounds (VIIa,b). A solution of Va,b $(0.01 \mathrm{~mol})$ in $15 \mathrm{~mL}$ of acetic anhydride was heated under reflux for one hour. The solution was then poured into $150 \mathrm{~mL}$ of ice-water and stirred for several hours until crystallization was complete. The precipitate was filtered off and washed with water then crystallized from an appropriate solvent to afford VIIa,b.

Ethyl 1-acetyl-2-(methylthio)-4,6-diphenyl-1,6-dihydropyrimidine-5-carboxylate (VIIa). The solid product crystallized from petroleum ether (60-80); yield (90\%); mp. $=102^{\circ} \mathrm{C}$; I.R $(\mathrm{KBr}): v=2978,1697,1601,1533 \mathrm{~cm}^{-1} ;{ }^{1} \mathrm{H}$ NMR $(300 \mathrm{MHz}$, $\mathrm{CDCl}_{3}$ ): $0.97\left(\mathrm{t}, J=6.6 \mathrm{~Hz}, 3 \mathrm{H}, \mathrm{CH}_{3} \mathrm{CH}_{2} \mathrm{O}-\right), 2.50(\mathrm{~s}, 3 \mathrm{H}$, $-\mathrm{SCH}_{3}$ ), 2.50 (s, 3H, $-\mathrm{COCH}_{3}$ ), 4.02 (q, $2 \mathrm{H}, \mathrm{CH}_{3} \mathrm{CH}_{2} \mathrm{O}-$ ), $6.66(\mathrm{~s}, 1 \mathrm{H},-\mathrm{CH}), 7.27-7.60(\mathrm{~m}, 10 \mathrm{H}, \mathrm{Ar}) \mathrm{ppm}$; mass: $m / z$ (\%): $394\left(\mathrm{M}^{+}, 12.51\right), 351$ (100.0), 337 (10.86), 323 (28.06), 275 (84.24), 129 (18.40), 77 (29.31); $\mathrm{C}_{22} \mathrm{H}_{22} \mathrm{~N}_{2} \mathrm{O}_{3} \mathrm{~S}$ (394): calculated, \%: C 66.98, H 5.62, N 7.10, O 12.17, S 8.13; found, \%: C 66.97, H 5.63, N 7.09, O 12.18, S 8.12.

1,1'-(6-(4-Methoxyphenyl)-4-methyl-2-(methylthio)pyrimidine-1,5(6H)-diyl)diethanone (VIIb). The precipitated product was crystallized from benzene; yield (86\%); mp. = $180^{\circ}$ C. I.R $(\mathrm{KBr}): v=2990,1675,1568 \mathrm{~cm}^{-1}$; mass: $\mathrm{m} / z$ 
(\%): $332\left(\mathrm{M}^{+}, 43.22\right), 312$ (39.56), 278 (51.28), 100 (100.0), 67 (50.55); $\mathrm{C}_{17} \mathrm{H}_{20} \mathrm{~N}_{2} \mathrm{O}_{3} \mathrm{~S}$ (332): calculated, \%: C 61.42, H 6.06, $\mathrm{N} 8.43$, O 14.44, S 9.65; found, \%: C 61.43, H 6.04, N 8.46, O $14.45, \mathrm{~S} 9.66$.

Synthesis of Ethyl 1-Methyl-2-(methylthio)-4,6-diphenyl-1,6dihydro-pyrimidine-5-carboxylate (VIIIa). A mixture of Va $(0.005 \mathrm{~mol})$ and methyl iodide $(0.005 \mathrm{~mol})$ was dissolved in DMF in the presence of pot. Carbonate anhydrous was refluxed in water bath for 4 hours. The reaction mixture was filtered on hot then the filtrate was cooled and poured onto cold water with stirring; the formed solid was filtered off and crystallized from ethanol: benzene $(3: 1) ; \mathrm{mp} .=92^{\circ} \mathrm{C}$; I.R (KBr): $v=3058,2977,2932,1717,1580 \mathrm{~cm}^{-1} ;{ }^{1} \mathrm{H}$ NMR $\left(300 \mathrm{MHz}, \mathrm{CDCl}_{3}\right): \delta=0.84\left(\mathrm{t}, \mathrm{J}=6.9 \mathrm{~Hz}, 3 \mathrm{H},-\mathrm{O}-\mathrm{CH}_{2}-\right.$ $\left.\mathrm{CH}_{3}\right), 2.57$ (s, 3H, $-\mathrm{SCH}_{3}$ ), $3.33\left(\mathrm{~s}, 3 \mathrm{H},-\mathrm{NCH}_{3}\right), 4.01$ (q, $2 \mathrm{H}$, $-\mathrm{O}-\mathrm{CH}_{2}-\mathrm{CH}_{3}$ ), 5.74 (s, 1H, $\left.-\mathrm{CH}-\right), 7.49-7.64$ (m, 10H, Ar) ppm; mass: $m / z$ (\%): $366\left(\mathrm{M}^{+}, 5.21\right), 350$ (100.0), 321 (28.48), 129 (30.96), 77 (15.87); $\mathrm{C}_{21} \mathrm{H}_{22} \mathrm{~N}_{2} \mathrm{O}_{2} \mathrm{~S}$ (366): calculated, \%: C 68.82, H 6.05, N 7.64, O 8.73, S 8.75; found, \%: C 68.81, H 6.04, N 7.65, O 8.73, S 8.75; yield (25\%).

\subsubsection{General Procedure for the Preparation of Compounds (Xa,b)}

Method (A). A mixture of IVa,b (1 mmol) and chloroacetic acid (1 mmol) was dissolved in $40 \mathrm{~mL}$ of a mixture of $(\mathrm{AC})_{2} \mathrm{O} / \mathrm{ACOH}(1: 3)$ in the presence of $3 \mathrm{gm}$ anhydrous sodium acetate that was refluxed for 4 hours. The reaction mixture was cold and poured onto cold water with stirring; the solid formation was filtered off and crystallized from benzene/ethanol $(3: 1)$ to give $\mathbf{X a , b}$.

Method (B). A mixture of IVa,b (0.005 mol) and chloroacetyl chloride with 2 drops of T.E.A was refluxed in benzene for 3 hours. Then the reaction mixture was filtered off through heating then dried and crystallized from benzene/ethanol $(3: 1)$ to give $\mathbf{X a}, \mathbf{b}$.

Method (C). A mixture of IVa,b $(0.005 \mathrm{~mol})$, ethylchloro acetate $(0.005 \mathrm{~mol})$, and sodium acetate trihydrate $(1 \mathrm{gm})$ was refluxed in ethanol for 5 hours. The reaction mixture was filtered and kept for overnight. The solid formation was filtered off then dried and crystallized from benzene/ethanol 3/1 to afforded $\mathbf{X a , b .}$

Ethyl 3-oxo-5,7-diphenyl-3,5,8,8a-tetrahydro-2H-thiazolo[3, 2-a]pyrimidine-6-carboxylate (Xa). The formed solid was crystallized from benzene/ethanol (3:1); method (A): yield $(72 \%)$; method $(B)$ : yield (75\%); method $(C)$ : yield $(84 \%)$. $\mathrm{mp} .=136-8^{\circ} \mathrm{C}$; I.R $(\mathrm{KBr}): \nu=2976,2931,2900,1752,1675$, $1589 \mathrm{~cm}^{-1} ;{ }^{1} \mathrm{H}$ NMR $\left(300 \mathrm{MHz}, \mathrm{CDCl}_{3}\right): \delta=0.85(\mathrm{t}, J=$ $\left.6.9 \mathrm{~Hz}, 3 \mathrm{H},-\mathrm{O}-\mathrm{CH}_{2}-\mathrm{CH}_{3}\right), 3.85\left(\mathrm{q}, 2 \mathrm{H},-\mathrm{O}-\mathrm{CH}_{2}-\mathrm{CH}_{3}\right), 3.88$ (s, 2H, $\left.-\mathrm{CH}_{2} \mathrm{CO}-\right), 6.19$ (s, $\left.1 \mathrm{H},-\mathrm{CH}-\right), 7.27-7.51(\mathrm{~m}, 10 \mathrm{H}$, Ar) ppm; mass: $m / z(\%): 378\left(\mathrm{M}^{+}, 60.03\right), 350$ (16.30), 301 (100.0), 273 (35.62), 129 (25.11), 77 (35.57); $\mathrm{C}_{21} \mathrm{H}_{18} \mathrm{~N}_{2} \mathrm{O}_{3} \mathrm{~S}$ (378): calculated, \%: C 66.65, H 4.79, N 7.40, O 12.68, S 8.47; found, \%: C 66.65, H 4.78, N 7.41, O 12.67, S 8.47.
6-Acetyl-5-(4-methoxyphenyl)-7-methyl-8,8a-dihydro-2Hthiazolo[3,2-a]pyrimidin-3(5H)-one (Xb). The formed solid was crystallized from benzene and drops of ethanol; method (A): yield (42\%). method (B): yield (34\%). method $(C)$ : yield (40\%). mp. $=160-162^{\circ} \mathrm{C}$; I.R $(\mathrm{KBr}): \nu=2983,2936,2876$, 1756, 1655, $1612 \mathrm{~cm}^{-1} ;{ }^{1} \mathrm{H}$ NMR (300 MHz, DMSO): $\delta=2.16$ $\left(\mathrm{s}, 3 \mathrm{H}, \mathrm{CH}_{3}\right), 2.34\left(\mathrm{~s}, 3 \mathrm{H},-\mathrm{COCH}_{3}\right), 3.70\left(\mathrm{~s}, 3 \mathrm{H}, \mathrm{OCH}_{3}\right)$, 4.15 (s, 2H, - $\mathrm{CH}_{2} \mathrm{CO}-$ ), 5.98 (s, 1H, - CH-), 6.86-7.21 (d, d, $4 \mathrm{H}$, Ar) ppm; mass: $m / z(\%): 316\left(\mathrm{M}^{+}, 35.08\right), 301$ (5.32), 273 (100.0), 245 (28.08), 230 (4.72), 181 (19.36), 115 (25.14), 77 (26.0); $\mathrm{C}_{16} \mathrm{H}_{18} \mathrm{~N}_{2} \mathrm{O}_{3} \mathrm{~S}$ (316): calculated, \%: C 60.36, H 5.70, N 8.80, O 15.08, S 10.07; found, \%: C 60.35, H 5.70, N 8.81, O 15.06, S 10.08 .

5.1.6. Synthesis of Compounds (XIa,b,c). A mixture of compound $\mathbf{X a}(1 \mathrm{mmol})$, aromatic aldehyde $(1 \mathrm{mmol})$, and 2 drops of piperidine was refluxed in ethanol for 2 hours. The reaction mixture kept overnight, then the solid product was filtered off and crystallized from EtOH to afford compound XI.

(E)-Ethyl 2-(4-chlorobenzylidene)-3-oxo-5,7-diphenyl-3,5-dihydro-2H-thiazolo [3,2-a]pyrimidine-6-carboxylate (XIa). mp. $=164-6^{\circ} \mathrm{C}$; I.R $(\mathrm{KBr}): v=3446,1721,1620,1559 \mathrm{~cm}^{-1}$; ${ }^{1} \mathrm{H}$ NMR $\left(300 \mathrm{MHz}, \mathrm{CDCl}_{3}\right): \delta=0.87(\mathrm{t}, J=6.9 \mathrm{~Hz}, 3 \mathrm{H}$, $-\mathrm{O}-\mathrm{CH}_{2}-\mathrm{CH}_{3}$ ), 3.89 (q, $\left.2 \mathrm{H},-\mathrm{O}-\mathrm{CH}_{2}-\mathrm{CH}_{3}\right), 6.34(\mathrm{~s}, 1 \mathrm{H}$, - $\mathrm{CH}-$ ), 7.27-7.54 (m, 14H, Ar), 7.74 (s, 1H, =CH-) ppm; mass: $\mathrm{m} / z(\%): 500\left(\mathrm{M}^{+2}, 45.80\right), 503\left(\mathrm{M}^{+4}, 9.82\right), 423$ (67.99), 168 (100.0), 77 (77.69); $\mathrm{C}_{28} \mathrm{H}_{21} \mathrm{ClN}_{2} \mathrm{O}_{3} \mathrm{~S}$ (500): calculated, \%: C 67.13, H 4.22, Cl 7.08, N 5.59, O 9.58, S 6.40; found, \%: C 67.14, $\mathrm{H} 4.24, \mathrm{Cl} 7.07$, N 5.60, O 9.59, S 6.42; yield (88\%).

(E)-Ethyl 2-(2,3-dimethoxybenzylidene)-3-oxo-5,7-diphenyl3,5-dihydro-2H-thiazolo [3,2-a]pyrimidine-6-carboxylate (XIb). mp. $=148^{\circ} \mathrm{C}$; I.R $(\mathrm{KBr}): v=34454,1712,1630$, $1581 \mathrm{~cm}^{-1} ;{ }^{1} \mathrm{H}$ NMR $\left(300 \mathrm{MHz}, \mathrm{CDCl}_{3}\right): \delta=0.87(\mathrm{t}$, $\left.J=6.6 \mathrm{~Hz}, 3 \mathrm{H},-\mathrm{O}-\mathrm{CH}_{2}-\mathrm{CH}_{3}\right), 3.77\left(\mathrm{~s}, 3 \mathrm{H},-\mathrm{OCH}_{3}\right), 3.85$ (s, 3H, $\left.-\mathrm{OCH}_{3}\right), 6.34$ (s, 1H, -CH-), 6.84 (d, 1H Ar), 6.96 (s, 1H, Ar), 6.98 (d, 1H, Ar), 7.35-7.55 (m, 10H, Ar), 8.09 (s, 1H, =CH-) ppm; mass: $m / z(\%): 526\left(\mathrm{M}^{+1}, 42.98\right), 449$ (100.0), 363 (27.0), 77 (5.37); $\mathrm{C}_{30} \mathrm{H}_{26} \mathrm{~N}_{2} \mathrm{O}_{5} \mathrm{~S}$ (526): calculated, \%: C 68.42, H 4.98, N 5.32, O 15.19, S 6.09; found, \%: C 68.43, H 4.99, N 5.33, O 15.20, S 6.10; Yield (85\%).

(E)-Ethyl2-(3-nitrobenzylidene)-3-oxo-5,7-diphenyl-3,5-dihydro-2H-thiazolo[3,2-a]pyrimidine-6-carboxylate (XIc). mp. $=158-160^{\circ} \mathrm{C}$; I.R (KBr): $\nu=3100,2998,1716,1617,1557$, $1563,1530 \mathrm{~cm}^{-1} ;{ }^{1} \mathrm{H}$ NMR $\left(300 \mathrm{MHz}, \mathrm{CDCl}_{3}\right): \delta=0.84(\mathrm{t}$, $\left.J=6.9 \mathrm{~Hz}, 3 \mathrm{H},-\mathrm{O}-\mathrm{CH}_{2}-\mathrm{CH}_{3}\right), 3.86\left(\mathrm{q}, 2 \mathrm{H},-\mathrm{O}-\mathrm{CH}_{2}-\mathrm{CH}_{3}\right)$, 6.31 (s, 1H, -CH-), 7.20-7.75 (m, 14H, Ar), 8.31 (s, 1H, =CH-) ppm; $\mathrm{C}_{28} \mathrm{H}_{21} \mathrm{~N}_{3} \mathrm{O}_{5} \mathrm{~S}$ (511): calculated, \%: C 65.74, $\mathrm{H} 4.14, \mathrm{~N}$ 8.21, O 15.64, S 6.27; found, \%: C 65.75, H 4.13, N 8.22, O 15.63, S 6.28; yield (92\%).

Synthesis of 1-[5-Acetyl-6-(4-methoxy-phenyl)-4-methyl-1,6dihydro-pyrimidin-2-ylsulfanyl]-propan-2-one (XIII). A mixture of IVb $(0.005 \mathrm{~mol})$, chloroacetone $(0.005 \mathrm{~mol})$, and 2 drops of piperidine was refluxed in ethanol for 6 hours. The reaction mixture was kept for overnight. The solid formation was filtered off then dried and crystallized from 
benzene/ethanol $(1: 1)$; mp. $=215^{\circ} \mathrm{C}$; I.R $(\mathrm{KBr}): v=3112$, $3004,1644,1608,1524 \mathrm{~cm}^{-1}$; ${ }^{1} \mathrm{H}$ NMR (300 MHz, DMSO): $\delta=2.25\left(\mathrm{~s}, 3 \mathrm{H},-\mathrm{CH}_{3}\right), 2.26\left(\mathrm{~s}, 3 \mathrm{H},-\mathrm{COCH}_{3}\right), 2.30(\mathrm{~s}, 3 \mathrm{H}$, $\left.-\mathrm{COCH}_{3}\right), 2.46\left(\mathrm{~s}, 2 \mathrm{H}, \mathrm{CH}_{2}\right), 3.71\left(\mathrm{~s}, 3 \mathrm{H},-\mathrm{OCH}_{3}\right), 6.44(\mathrm{~s}$, $1 \mathrm{H},-\mathrm{CH}), 6.90-7.28$ (d, d, 4H, Ar), 7.11 (s, 1H, -NH); mass: $m / z$ (\%): $332\left(\mathrm{M}^{+}, 5.30\right), 298$ (5.30), 270 (33.60), 245 (25.7), 91 (100.0); $\mathrm{C}_{17} \mathrm{H}_{20} \mathrm{~N}_{2} \mathrm{O}_{3} \mathrm{~S}$ (332): calculated, \%: C 61.42, H 6.06, N 8.43, O 14.44, S 9.65; found, \%: C 61.43, H 6.05, N 8.43, O 14.42, S 9.66; yield (48\%).

5.1.7. Reaction of Compounds Va,b with Thiosemicarbazide. A mixture of Va,b $(0.005 \mathrm{~mol})$ and thiosemicarbazide $(0.07 \mathrm{~mol})$ was refluxed in ethanol $(20 \mathrm{~mL})$ for 7 hours in a water bath, then the reaction mixture was allowed to stand for several hours at room temperature, then the solid product was filtered off and recrystallized from ethanol to formed compounds XIVa,b.

Ethyl 2-(2-carbamothioylhydrazinyl)-4,6-diphenyl-1,6-dihydro-pyrimidine-5-carboxylate (XIVa). Method (A): yield (51\%); method (B): yield (46\%); mp. $=170-172^{\circ} \mathrm{C} \mathrm{I} \cdot \mathrm{R}(\mathrm{KBr})$ : $v=3370,3262,3175,1644,1620 \mathrm{~cm}^{-1} .{ }^{1} \mathrm{H}$ NMR $(300 \mathrm{MHz}$, DMSO): $\delta=0.71\left(\mathrm{t}, J=7.5 \mathrm{~Hz}, 3 \mathrm{H}, \mathrm{OCH}_{2} \mathrm{CH}_{3}\right), 3.74$ $\left(\mathrm{q}, 2 \mathrm{H}, \mathrm{OCH}_{2} \mathrm{CH}_{3}\right), 4.50\left(\mathrm{~s}, 2 \mathrm{H},-\mathrm{NH}_{2}\right), 5.26(\mathrm{~s},-\mathrm{CH})$, 7.19-7.58 (m, 10H, Ar), $8.65(\mathrm{~s}, 1 \mathrm{H},-\mathrm{NH}), 9.78$ (s, 1H, $\mathrm{NH}), 10.51$ (s, $1 \mathrm{H},-\mathrm{NH})$ ppm; mass: $\mathrm{m} / z(\%): 395\left(\mathrm{M}^{+}\right.$, 24.13), 379 (21.13), 368 (49.20), 352 (76.97), 105 (86.51), 55 (100.0); $\mathrm{C}_{20} \mathrm{H}_{21} \mathrm{~N}_{5} \mathrm{O}_{2} \mathrm{~S}$ (395): calculated, \%: C 60.74, H 5.35, N 17.71, O 8.09, S 8.11; found, \%: C 60.73, H 5.36, N 17.71, O 8.08.

2-(5-Acetyl-6-(4-methoxyphenyl)-4-methyl-1,6-dihydropyrimidin-2-yl)hydrazinecarbothioamide (XIVb). Method (A): yield (64\%); method (B): yield $(48 \%) ; \mathrm{mp} .=92^{\circ} \mathrm{C}$; I.R $(\mathrm{KBr}): v=3284,3145,2049,1604,1510 \mathrm{~cm}^{-1} ;$ mass: $\mathrm{m} / z$ (\%): $333\left(\mathrm{M}^{+}, 12.18\right), 274$ (14.92), 233 (27.31), 215 (84.93), 178 (90.61), 136 (100.0), 76 (65.0), 51 (23.07); $\mathrm{C}_{15} \mathrm{H}_{19} \mathrm{~N}_{5} \mathrm{O}_{2} \mathrm{~S}$ (333): calculated, \%: C 54.04, H 5.74, N 21.01, O 9.60, S 9.62; found, \%: C 54.05, H 5.74, N 21.0, O 9.61, S 9.62.

5.1.8. Synthesis of Compounds XVIIa,b. Compound IVa,b $(0.005 \mathrm{~mol})$ was heated under reflux in $(10 \mathrm{~mL})$ of methanol, containing acetic acid $(2.5 \mathrm{~mL})$ and water $(2.5 \mathrm{~mL})$. After reflux for 25 hours, methanol was distilled off and the remaining solution was treated portionwise with water until precipitation was completed. After standing for several hours at room temperature, the solid product was removed by filteration to yield XVIIa,b which crystallized from ethanol.

Ethyl 2-oxo-4,6-diphenyl-1,2,3,4-tetrahydropyrimidine-5-carboxylate $($ XVIIa). mp. (found $)=160^{\circ} \mathrm{C}, \mathrm{mp}$. $($ reported $)=$ $158^{\circ} \mathrm{C}[48]$; yield $(77 \%)$.

5-Acetyl-4-(4-methoxyphenyl)-6-methyl-3,4-dihydropyrimidin-2(1H)-one (XVIIb). mp. (found) $=164-166^{\circ} \mathrm{C} ; \mathrm{mp}$. $($ reported $)=166-167^{\circ} \mathrm{C}[48]$; yield $(71 \%)$.

\section{References}

[1] E. W. Hurst and R. Hull, “Two new synthetic substances active against viruses of the psittacosis-lymphogranuloma- trachoma group," Journal of Medicinal Chemistry, vol. 3, no. 2, pp. 215-229, 1961.

[2] M. Ashok, B. S. Holla, and N. S. Kumari, "Convenient one pot synthesis of some novel derivatives of thiazolo[2,3$b$ ]dihydropyrimidinone possessing 4-methylthiophenyl moiety and evaluation of their antibacterial and antifungal activities," European Journal of Medicinal Chemistry, vol. 42, no. 3, pp. 380 385, 2007.

[3] S. S. Bahekar and D. B. Shinde, "Synthesis and anti-inflammatory activity of some [4,6-(4-substituted aryl)-2-thioxo-1,2,3,4tetrahydro-pyrimidin-5-yl]-acetic acid derivatives," Bioorganic \& Medicinal Chemistry Letters, vol. 14, no. 7, pp. 1733-1736, 2004.

[4] T. U. Mayer, T. M. Kapoor, S. J. Haggarty, R. W. King, S. L. Schreiber, and T. J. Mitchison, "Smart molecule inhibitor of mitotic spindle bipolarity identified in a phenotype-based screen," Science, vol. 286, no. 5441, pp. 971-974, 1999.

[5] C. O. Kappe, "Recent advances in the Biginelli dihydropyrimidine synthesis. New tricks from an old dog," Accounts of Chemical Research, vol. 33, no. 12, pp. 879-888, 2000.

[6] A. D. Patil, N. V. Kumar, W. C. Kokke et al., "Novel alkaloids from the sponge Batzella sp.: inhibitors of HIV gp120-human CD4 binding," Journal of Organic Chemistry, vol. 60, no. 5, pp. 1182-1188, 1995.

[7] B. B. Snider, J. Chen, A. D. Patil, and A. J. Freyer, "Synthesis of the tricyclic portions of batzelladines A, B and D. Revision of the stereochemistry of batzelladines A and D," Tetrahedron Letters, vol. 37, no. 39, pp. 6977-6980, 1996.

[8] P. Biginelli, "Derivati aldeidureidici degli eteri acetil-ed ossalacetico," Gazzetta Chimica Italiana, vol. 23, pp. 360-416, 1893.

[9] P. Wipf and A. Cunningham, "A solid phase protocol of the Biginelli dihydropyrimidine synthesis suitable for combinatorial chemistry," Tetrahedron Letters, vol. 36, no. 43, pp. 7819-7822, 1995.

[10] C. O. Kappe, "100 years of the Biginelli dihydropyrimidine synthesis," Tetrahedron, vol. 49, no. 32, pp. 6937-6963, 1993.

[11] D. Dallinger, A. Stadler, and C. O. Kappe, "Solid- and solutionphase synthesis of bioactive dihydropyrimidines," Pure and Applied Chemistry, vol. 76, no. 5, pp. 1017-1024, 2004.

[12] C. O. Kappe, "4-aryldihydropyrimidines via the Biginelli condensation: aza-analogs of nifedipine-type calcium channel modulators," Molecules, vol. 3, no. 1, pp. 1-9, 1998.

[13] C. O. Kappe and A. Stadler, "The Biginelli dihydropyrimidine synthesis," Organic Reactions, vol. 63, pp. 1-116, 2004.

[14] C. Simon, T. Constantieux, and J. Rodriguez, "Utilisation of 1,3dicarbonyl derivatives in multicomponent reactions," European Journal of Organic Chemistry, no. 24, pp. 4957-4980, 2004.

[15] C. O. Kappe, "The generation of dihydropyrimidine libraries utilizing Biginelli multicomponent chemistry," QSAR and Combinatorial Science, vol. 22, no. 6, pp. 630-645, 2003.

[16] H. E. Zaugg and W. B. Martin, " $\alpha$-amidoalkylations at carbon," in Organic Reactions, vol. 14, pp. 88-90, John Wiley \& Sons, New York, NY, USA, 1965.

[17] C. O. Kappe, "The Biginelli reaction," in Multicomponent Reactions, pp. 95-120, John Wiley \& Sons, New York, NY, USA, 2005. 
[18] L. Z. Gong, X. H. Chen, and X. Y. Xu, "Asymmetric organocatalytic biginelli reactions: a new approach to quickly access optically active 3,4-dihydropyrimidin-2-(1H)-ones," Chemistry-A European Journal, vol. 13, no. 32, pp. 8920-8926, 2007.

[19] A. Dondoni and A. Massi, "Parallel synthesis of dihydropyrimidinones using $\mathrm{Yb}(\mathrm{III})$-resin and polymer-supported scavengers under solvent-free conditions. A green chemistry approach to the Biginelli reaction," Tetrahedron Letters, vol. 42, no. 45, pp. 7975-7978, 2001.

[20] A. Shaabani and A. Bazgir, "Microwave-assisted efficient synthesis of spiro-fused heterocycles under solvent-free conditions," Tetrahedron Letters, vol. 45, no. 12, pp. 2575-2577, 2004.

[21] B. J. Ahn, M. S. Gang, K. Chae, Y. Oh, J. Shin, and W. Chalg, "A microwave-assisted synthesis of 3,4-dihydro-pyrimidin-2(1H)-ones catalyzed by $\mathrm{FeCl}_{3}$-supported Nanopore Silica under solvent-free conditions," Journal of Industrial and Engineering Chemistry, vol. 14, no. 3, pp. 401-405, 2008.

[22] X. Zhang, Y. Li, C. Liu, and J. Wang, "An efficient synthesis of 4-substituted pyrazolyl-3,4-dihydropyrimidin-2(1H)(thio)ones catalyzed by $\mathrm{Mg}\left(\mathrm{ClO}_{4}\right)_{2}$ under ultrasound irradiation," Journal of Molecular Catalysis A, vol. 253, no. 1-2, pp. 207211, 2006.

[23] J. T. Li, J. F. Han, J. H. Yang, and T. S. Li, "An efficient synthesis of 3,4-dihydropyrimidin-2-ones catalyzed by $\mathrm{NH}_{2} \mathrm{SO}_{3} \mathrm{H}$ under ultrasound irradiation," Ultrasonics Sonochemistry, vol. 10, no. 3, pp. 119-122, 2003.

[24] N. Foroughifar, A. Mobinikhaledi, and H. Fathinejad Jirandehi, "Synthesis of some biginelli compounds in solvent medium using a photochemistry method," Phosphorus, Sulfur and Silicon and the Related Elements, vol. 178, no. 3, pp. 495-500, 2003.

[25] E. H. Hu, D. R. Sidler, and U. H. Dolling, "Unprecedented catalytic three component one-pot condensation reaction: an efficient synthesis of 5-alkoxycarbonyl-4-aryl-3,4dihydropyrimidin- 2(1H)-ones," Journal of Organic Chemistry, vol. 63, no. 10, pp. 3454-3457, 1998.

[26] C. V. Reddy, M. Mahesh, P. V. K. Raju, T. R. Babu, and V. V. N. Reddy, "Zirconium(IV) chloride catalyzed one-pot synthesis of 3,4-dihydropyrimidin-2(1H)-ones," Tetrahedron Letters, vol. 43, no. 14, pp. 2657-2659, 2002.

[27] A. Kumar and R. A. Maurya, "An efficient bakers' yeast catalyzed synthesis of 3,4-dihydropyrimidin-2-(1H)-ones," Tetrahedron Letters, vol. 48, no. 26, pp. 4569-4571, 2007.

[28] Y. Q. Zhang, C. Wang, G. S. Li, J. C. Li, H. M. Liu, and Q. H. Wu, "One-pot Synthesis of 3,4-Dihydropyrimidin-2(1H)-ones Catalyzed by Expandable Graphite," Chinese Journal of Organic Chemistry, vol. 25, pp. 1265-1267, 2005.

[29] R. V. Yarapathi, S. Kurva, and S. Tammishetti, "Synthesis of 3,4-dihydropyrimidin-2(1H)ones using reusable poly(4vinylpyridine-co-divinylbenzene)-Cu(II)complex," Catalysis Communications, vol. 5, no. 9, pp. 511-513, 2004.

[30] J. Azizian, A. A. Mohammadi, A. R. Karimi, and M. R. Mohammadizadeh, " $\mathrm{KAl}\left(\mathrm{SO}_{4}\right)_{2} \cdot 12 \mathrm{H}_{2} \mathrm{O}$ supported on silica gel as a novel heterogeneous system catalyzed biginelli reaction: one-pot synthesis of di-hydropyrimidinones under solvent-free conditions," Applied Catalysis A, vol. 300, no. 1, pp. 85-88, 2006.

[31] N. Mizuno and M. Misono, "Heterogeneous catalysis," Chemical Reviews, vol. 98, no. 1, pp. 199-217, 1998.

[32] A. A. Bakibaev and V. D. Filimonov, "Synthesis of hydrogenated acridine-1,8-diones \& 1,4-dihydropyrimidines by reaction of urea with 1,3-dicarbonyl compounds," Russian Journal of Organic Chemistry, vol. 27, pp. 854-859, 1991.
[33] Y. Yu, D. Liu, C. Liu, and G. Luo, "One-pot synthesis of 3,4-dihydropyrimidin-2(1H)-ones using chloroacetic acid as catalyst," Bioorganic \& Medicinal Chemistry Letters, vol. 17, no. 12, pp. 3508-3510, 2007.

[34] A. S. Paraskar, G. K. Dewkar, and A. Sudalai, "Cu(OTf $)_{2}$ : a reusable catalyst for high-yield synthesis of 3,4-dihydropyrimidin-2(1H)-ones," Tetrahedron Letters, vol. 44, no. 16, pp. 3305$3308,2003$.

[35] M. Gohain, D. Prajapati, and S. Sandhub, "A novel Cu-catalysed three-component one-pot synthesis of dihydropyrimidin$2(1 H)$-ones using microwaves under solvent free conditions," Synlett, p. 235, 2004.

[36] Y. Ma, C. Qian, L. Wang, and M. Yang, "Lanthanide triflate catalyzed Biginelli reaction. one-pot synthesis of dihydropyrimidinones under solvent-free conditions," The Journal of Organic Chemistry, vol. 65, no. 12, pp. 3864-3868, 2000.

[37] V. T. Kamble, D. B. Muley, S. T. Atkore, and S. D. Dakore, "Three component reaction: an efficient synthesis of 3,4dihydropyrimidin-2(1H)-ones and thiones using heterogeneous catalyst," Chinese Journal of Chemistry, vol. 28, no. 3, pp. 388-392, 2010.

[38] B. Gangadasu, S. Palaniappan, and V. J. Rao, "One-pot synthesis of dihydropyrimidinones using polyaniline-bismoclite complex. A facile and reusable catalyst for the biginelli reaction," Synlett, no. 7, pp. 1285-1287, 2004.

[39] A. K. Mitra and K. Banerjee, "Clay catalysed synthesis of dihydropyrimidinones under solvent-free conditions," Synlett, no. 10, pp. 1509-1511, 2003.

[40] J. Mabry and B. Ganem, "Studies on the Biginelli reaction: a mild and selective route to 3,4-dihydropyrimidin-2(1H)-ones via enamine intermediates," Tetrahedron Letters, vol. 47, no. 1, pp. 55-56, 2006.

[41] T. Ghorge, R. Tahilramani, and D. V. Mehta, "Condensed heterocycles from 5-ethoxycarbonyl-6-methyltetrahydropyrimidin-2-ones," Communications, pp. 405-407, 1975.

[42] N.-Y. Fu, Y.-F. Yuan, Z. Cao, S.-W. Wang, J.-T. Wang, and C. Peppe, "Indium(III) bromide-catalyzed preparation of dihydropyrimidinones: improved protocol conditions for the Biginelli reaction," Tetrahedron, vol. 58, no. 24, pp. 4801-4807, 2002.

[43] J. S. Yadav, B. V. S. Reddy, P. Sridhar et al., "Green protocol for the biginelli three-component reaction: $\mathrm{Ag} 3 \mathrm{PW}_{12} \mathrm{O}_{40}$ as a novel, water-tolerant heteropolyacid for the synthesis of 3,4dihydropyrimidinones," European Journal of Organic Chemistry, no. 3, pp. 552-557, 2004.

[44] K. Folkers and T. B. Johnson, "Researches on pyrimidines. CXXXVI. The mechanism of formation of tetrahydropyrimidines by the Biginelli reaction," Journal of the American Chemical Society, vol. 55, no. 9, pp. 3784-3791, 1933.

[45] R. D. Carlos, D. Bernardi, and G. Kirsch, " $\mathrm{ZrCl}_{4}$ or $\mathrm{ZrOCl}_{2}$ under neat conditions: optimized green alternatives for the Biginelli reaction," Tetrahedron Letters, vol. 48, no. 33, pp. 57775780, 2007.

[46] N. Foroughifar, A. Mobinikhaledi, H. F. Jirandehi, and S. Memar, "Microwave-assisted synthesis of some BI- and tricyclic pyrimidine derivatives," Phosphorus, Sulfur and Silicon and the Related Elements, vol. 178, no. 6, pp. 1269-1276, 2003.

[47] B. Ahmed, R. A. Khan, Habibullah, and M. Keshari, "An improved synthesis of Biginelli-type compounds via phasetransfer catalysis," Tetrahedron Letters, vol. 50, no. 24, pp. 28892892, 2009. 
[48] E. Ramu, V. Kotra, N. Bansal, R. Varala, and S. R. Adapa, “Green approach for the efficient synthesis of Biginelli compounds promoted by citric acid under solvent-free conditions," RASĀYAN Journal of Chemistry, vol. 1, no. 1, pp. 188-194, 2008. 

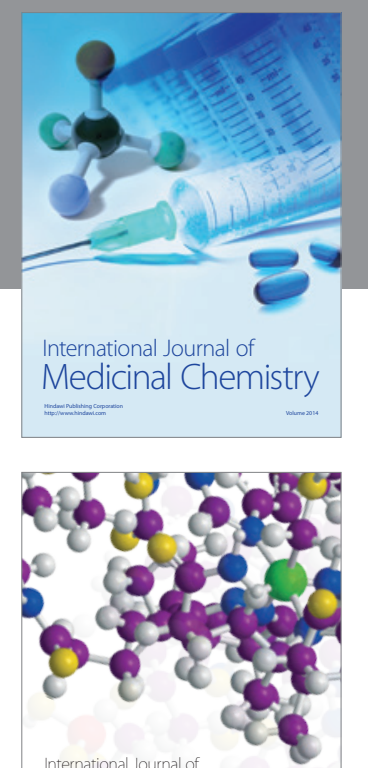

\section{Carbohydrate} Chemistry

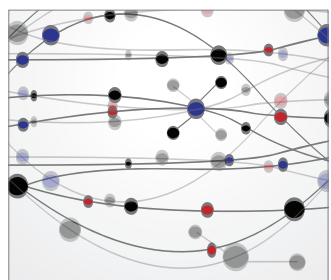

The Scientific World Journal
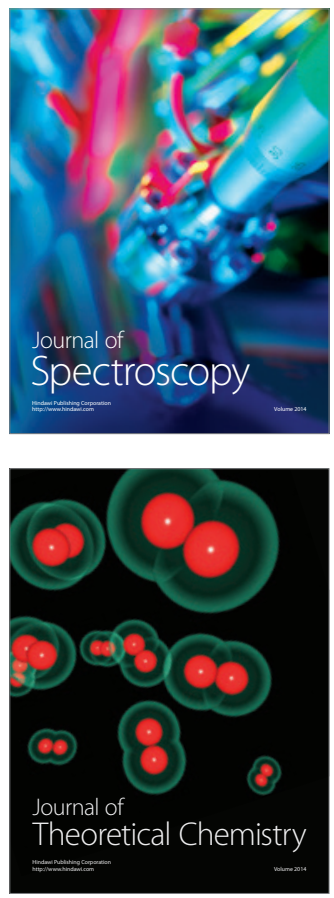
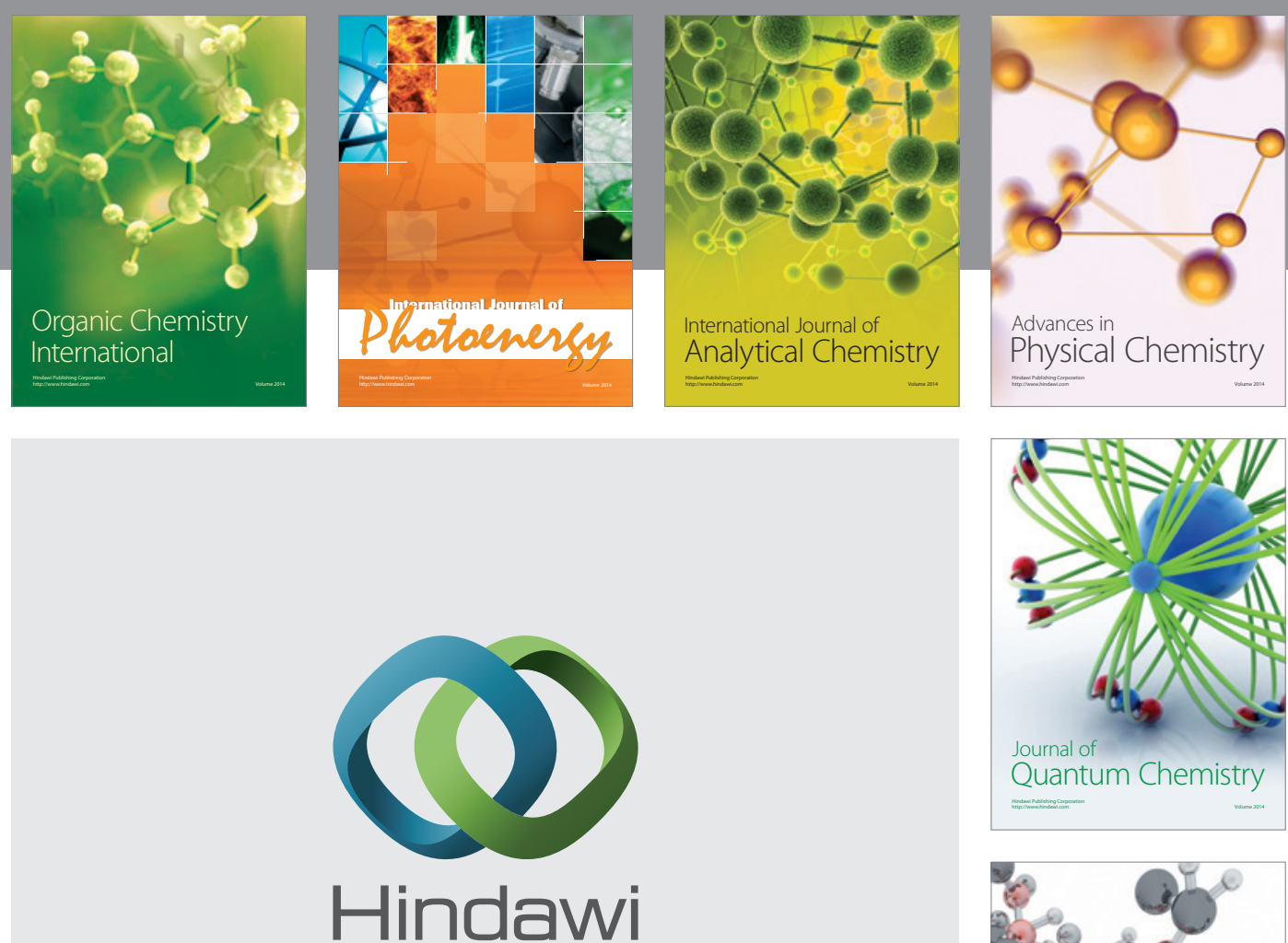

Submit your manuscripts at

http://www.hindawi.com

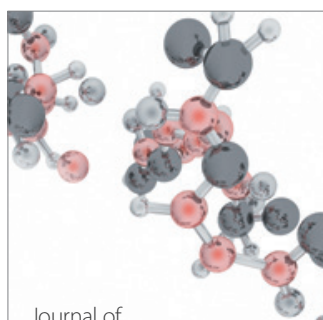

Analytical Methods

in Chemistry

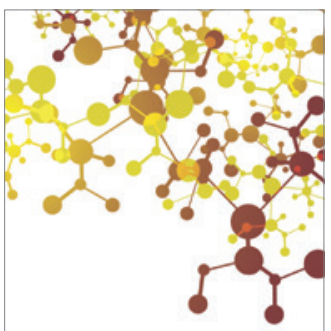

Journal of

Applied Chemistry

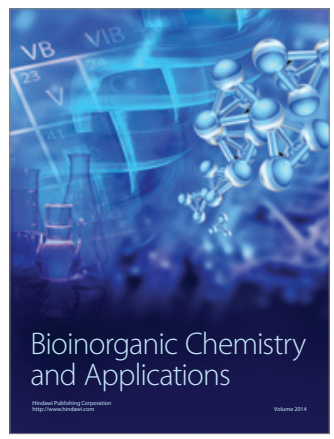

Inorganic Chemistry
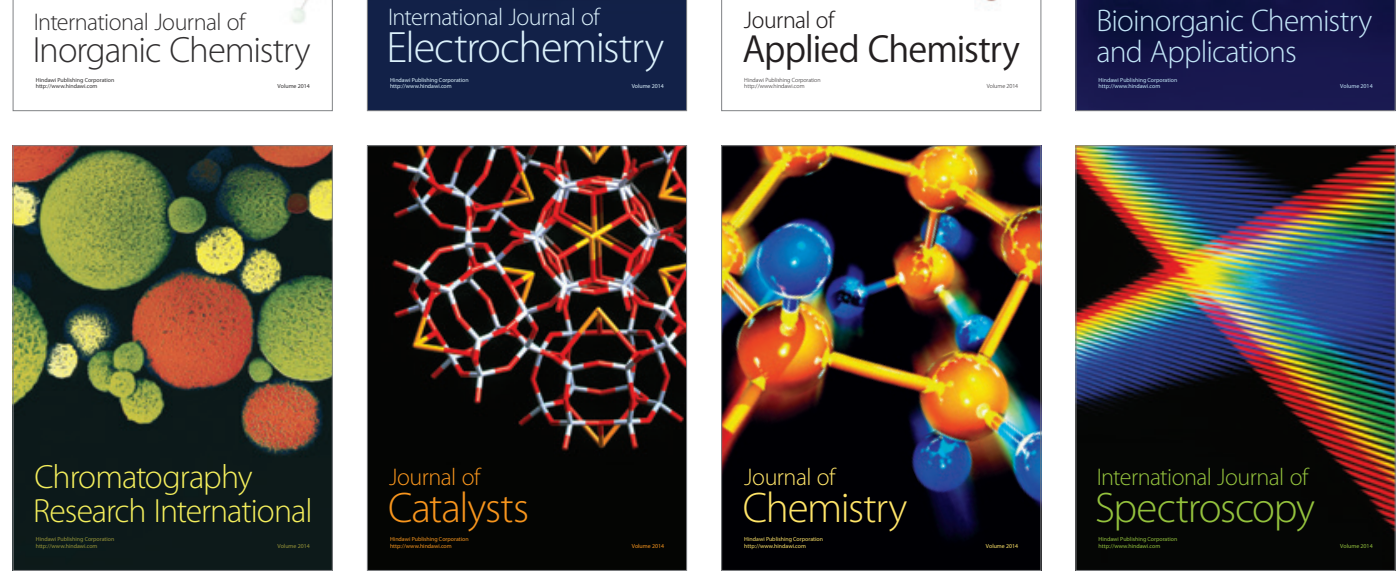